
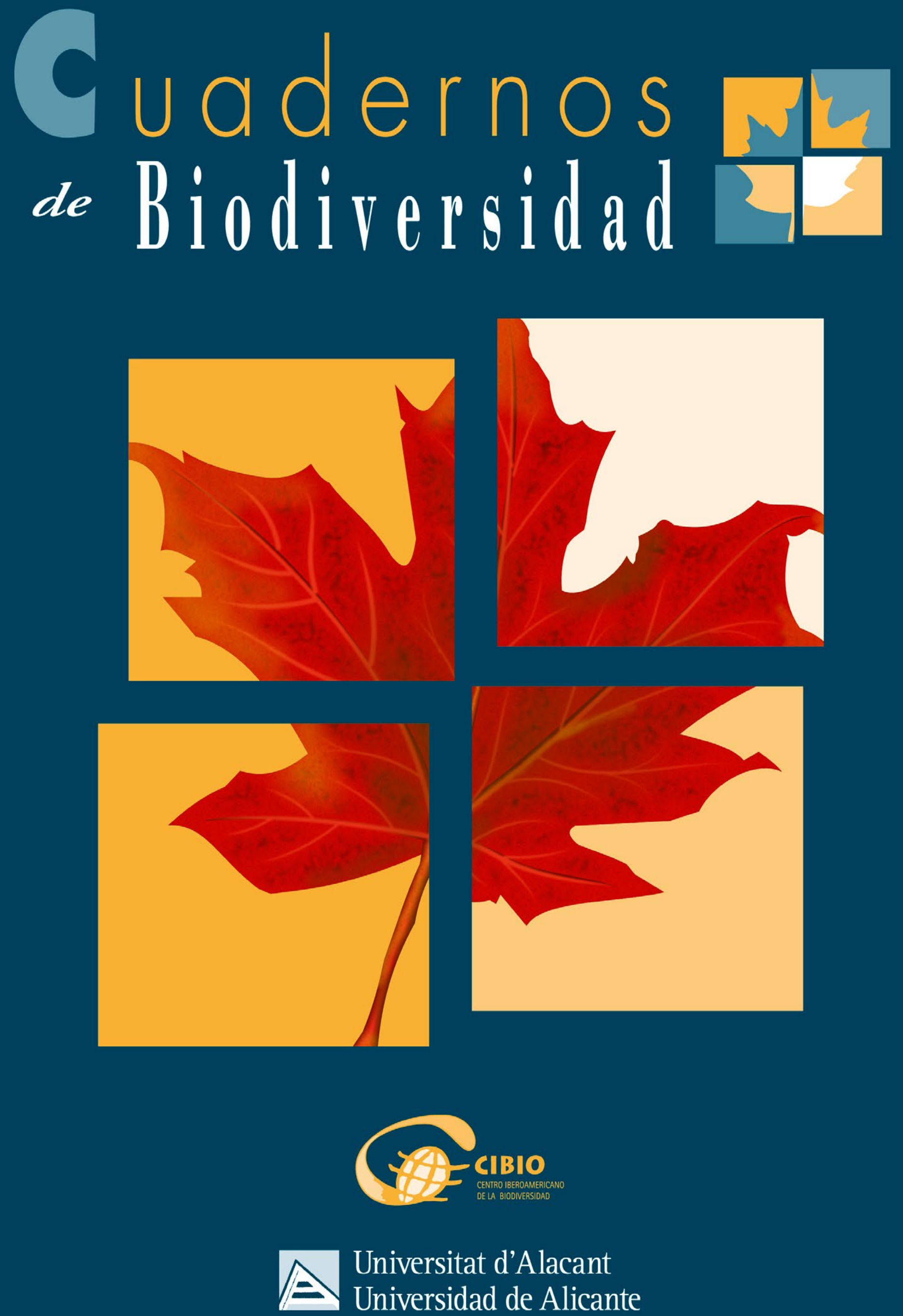


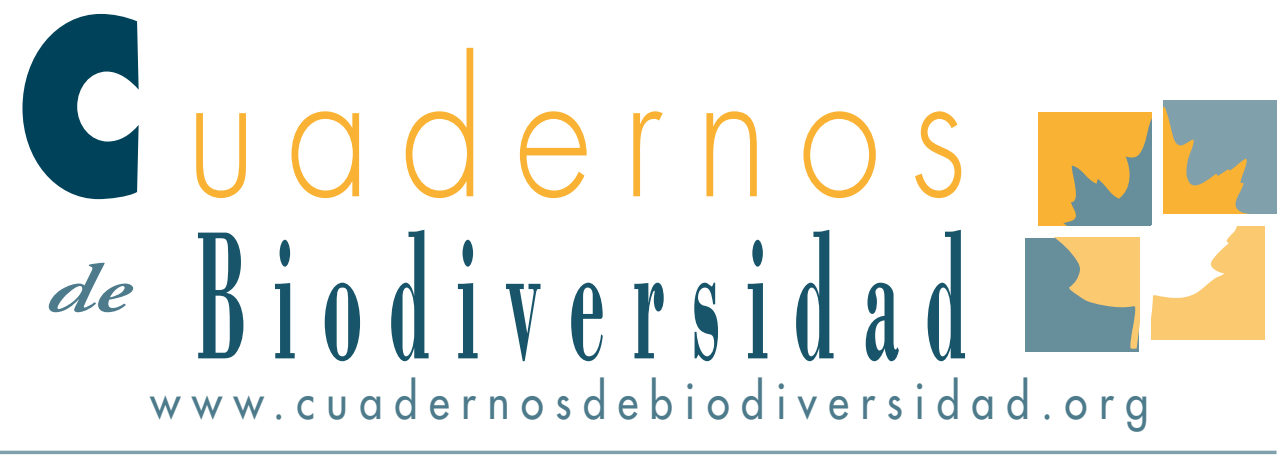

\section{Factores claves que afectan a la preferencia trófica y diversidad de coleópteros copro-necrófagos del bosque Atlántico de Argentina: una propuesta de conservación}

Key factors affecting trophic preferences and diversity of dung beetles in the Atlantic forest of Argentina: a conservation proposal

\section{V.C. Giménez GómeZ ${ }^{1}$, J.R. Verdú y G.A. Zurita ${ }^{\mathrm{I}, 3}$}

1 Instituto de Biología Subtropical- Universidad Nacional de Misiones - CONICET, Bertoni 85, Puerto Iguazú, Misiones (3370), Argentina.

2 I.U.I. CIBIO, Universidad de Alicante, Carretera de San Vicente del Raspeig s/n, Alicante 03690, España.

3 Facultad de Ciencias Forestales. Universidad Nacional de Misiones. Bertoni 128. Eldorado, Misiones. 


\section{RESUMEN}

La sustitución de ecosistemas naturales es una de las principales causas de pérdida de biodiversidad, ya que modifica las condiciones climáticas y recursos alimenticios para los organismos. En este trabajo se muestran los resultados de dos estudios ya publicados en el Bosque Atlántico de Argentina, uno asociado con el efecto de la perturbación de ecosistemas naturales sobre las preferencias tróficas de coleópteros copro-necrófagos y el otro asociado con el efecto de diferentes factores (presencia/ ausencia de cobertura de dosel arbóreo, presencia/ ausencia de ganado y vegetación nativa o exótica) sobre su riqueza y composición. En ambos estudios los coleópteros copro-necrófagos fueron muestreados en bosque nativo y cuatro usos de la tierra. Los resultados muestran que la perturbación ambiental afecta, en parte, a la preferencia trófica de coleópteros copro-necrófagos, ya que algunos tienden a tolerar las nuevas condiciones tróficas mientras que otros no, y que la pérdida de cobertura de dosel arbóreo es el factor principal que afecta su diversidad. La conclusión de este trabajo es que la perturbación antrópica afecta las preferencias tróficas y la diversidad de coleópteros copro-necrófagos, sobre todo en aquellos ambientes que pierden la cobertura de dosel arbóreo, por lo que su mantenimiento es la principal recomendación de manejo sugerida en este trabajo.

Palabras claves: cobertura de dosel arbóreo, nicho ecológico, ambiente de bosque, ganado, condiciones climáticas, Scarabaeoidea.

\section{ABSTRACT}

The replacement of natural ecosystems is one of the main causes of biodiversity loss, since it modifies climatic conditions and food resources for organisms. This work presents the results of two previous studies performed in the Atlantic Forest of Argentina, one evaluating the influence of human disturbance on dung beetles trophic preferences and the other dealing with the effects of different factors (presence/absence of canopy cover, presence/absence livestock and native or exotic vegetation) on dung beetles diversity (both richness and composition). In both studies dung beetles were sampled in native forest and under four land uses. The results show that environmental disturbance affects the trophic preference of dung beetles; some species were able to tolerate and exploit new resources while others became locally extinct. The second main result was that the absence of canopy is the main factor impacting dung beetles diversity. Human disturbance affects trophic preferences and the diversity of dung beetles, particularly in those land uses where the canopy was eliminated, therefore their conservation is the main recommendation suggested in this work.

Key words: canopy cover, ecological niche, forest habitat, livestock, microclimatic conditions, Scarabaeoidea.

\section{INTRODUCCIÓN}

En las últimas décadas, la alteración y sustitución de los ecosistemas naturales ha sido identificada como la principal causa de pérdida de diversidad (Dirzo y Raven, 2003). Particularmente, la expansión de la ganadería y agricultura ha tenido un gran efecto sobre la biodiversidad, particularmente en regiones de alta biodiversidad como los bosques tropicales y subtropicales (Davies y Margules, 1998; Myers et al., 2000; Myers y Knoll, 2001; Novacek y Cleland, 2001; Arellano et al., 2008). La degradación y reemplazo de los ecosistemas naturales cambia las condiciones ambientales (microclima, estructura del suelo) (Osberg et al., 1994; Oliveira-Filho y Fontes, 2000; Broennimann et al., 2012) y la disponibilidad espacial - temporal de recursos tróficos para los individuos (Culot et al., 2013). La magnitud de estos cambios afecta, en mayor o menor medida, la abundancia y diversidad de las especies (Halffter y Arellano, 2002; Andrade-Núñez y Aide, 2010). En general se ha observado que aquellos ambientes perturbados que conservan la estructura y composición de la vegetación, así como también las condiciones ambientales con respecto al ambiente natural (teoría de la similitud ambiental), son más utilizados por las especies nativas (Zurita y Bellocq, 2012; Audino et al., 2014; Gómez-Cifuentes et al., 2017; 2018). 
Los coleópteros copro-necrófagos (pertenecientes a los Scarabaeinae) son un excelente grupo para estudiar la influencia de las perturbaciones antrópicas (Verdú et al., 2007b; Gardner et al., 2008, Tonelli, 2017), sobre las poblaciones, comunidades y procesos ecosistémicos, por su abundancia y diversidad (Ocampo y Hawks, 2006; Spector, 2006), taxonomía relativamente estable (Philips et al., 2004) e importancia en el funcionamiento de los ecosistemas (Hanski y Cambefort, 1991; Andresen y Feer, 2005; Nichols et al., 2008; Verdú et al., 2018). Respecto a esto último, se destaca el enterramiento de materia orgánica (Davis, 1996 ), la dispersión secundaria de semillas, el reciclaje de nutrientes, el aumento de la aireación del suelo, el control de plagas y la reducción de gases con efecto invernadero (Andresen, 2003; Gardner et al., 2008; Slade et al., 2016).

Estudios previos realizados en el Bosque Atlántico (Hernández et al., 2014; Bogoni et al., 2014; Gómez-Cifuentes et al., 2017) y otros bosques tropicales y subtropicales (Vulinec, 2002; Quintero y Roslin, 2005; Nichols et al., 2007; Alvarado et al., 2018) han puesto de manifiesto que el reemplazo del ambiente natural provoca cambios tanto a nivel de población como de comunidad en coleópteros copro-necrófagos. Estos cambios generalmente se asocian a cambios en la estructura de la vegetación, condiciones microclimáticas, estructura del suelo y disponibilidad de recursos. Los cambios en la estructura de la vegetación tienen un efecto indirecto en la diversidad de coleópteros copro-necrófagos a través de las alteraciones en las condiciones microclimáticas, entre las que se destaca la radiación solar (Halffter et al., 1992; Verdú et al., 2007a), la intensidad de luz, la temperatura y humedad ambiental y del suelo (Davis et al., 2002).

En este grupo, en general, se ha observado que las especies de bosque nativo presentan restricciones fisiológicas y la mayoría de las especies son sensibles a los cambios en las condiciones microclimáticas (Davis et al., 2000; Duncan y Byrne, 2000, Giménez Gómez et al., comunicación personal). Respecto a los cambios en la estructura del suelo, éstos imponen nuevas restricciones para las especies de bosques nativos, ya que las mismas dependen de las condiciones de suelo (compactación, $\mathrm{pH}$, humedad relativa) para su nidificación (Osberg et al., 1994; Sowig, 1995; Nichols et al., 2013). Finalmente, hay estudios que muestran como las alteraciones del medio tienen un efecto indirecto sobre la diversidad de coleópteros copro-necrófagos asociado con la pérdida de grandes mamíferos, ya que estos coleópteros se alimentan principalmente del estiércol de estos animales (Nichols et al., 2009). En todos los casos, si bien cada especie responde de forma diferencial, de acuerdo a su nicho trófico, el impacto negativo sobre la biodiversidad es muy significativo (Christie y Hochuli, 2008). Hasta el momento, muchos estudios han mostrado el efecto de estas alteraciones del medio sobre la diversidad de especies, pero ninguno había mostrado el efecto sobre las preferencias tróficas de los individuos.

El Bosque Atlántico es uno de los ecosistemas más amenazados y con mayor diversidad biológica del mundo (Myers et al., 2000; Di Bitetti et al., 2003; Ribeiro et al., 2009). Se extiende desde una latitud tropical en los estados de Ceará y Río Grande del Norte en la costa noreste de Brasil, hasta una latitud subtropical más estacional en el estado sureño de Río Grande del Sur en Brasil, desde el Océano Atlántico hacia el interior, pasando sobre la cadena montańosa costera de Brasil y llegando hasta la cuenca del Río Paraná en el este de Paraguay y la provincia de Misiones en Argentina (Di Bitetti et al., 2003). Originalmente ocupaba alrededor de 150 millones de hectáreas (Ribeiro et al., 2009) distribuidas en estos tres países y actualmente ha perdido más del $92 \%$ de esta superficie original debido, principalmente, al reemplazo del bosque nativo por diferentes usos de la tierra (Di Bitetti et al., 2003; Holz y Placci, 2003; De Angelo, 2009). Entre los usos de la tierra que predominan en este ambiente se destacan las plantaciones de árboles exóticos, áreas abiertas con ganado y áreas de agricultura. Dada esta heterogeneidad de ambientes, el Bosque Atlántico es considerado un área de estudio adecuada para evaluar los cambios de diversidad provocados por las perturbaciones de origen antrópico (Izquierdo et al., 2008; Zurita y Bellocq, 2012). 
Teniendo en cuenta la importancia de los coleópteros copro-necrófagos y del Bosque Atlántico, se llevaron a cabo dos estudios en esta área, que muestran como las perturbaciones de origen antrópico afectan a las preferencias tróficas de las especies (primer estudio) y su riqueza y composición (segundo estudio). En el primer estudio, entre otros objetivos, se pretendía estudiar las preferencias tróficas (como componente del nicho trófico) de coleópteros copro-necrófagos en cada ambiente para saber si los cambios en diversidad se encontraban asociados a restricciones del nicho trófico de las especies. Por otro lado, en el segundo estudio, se pretendía estudiar el efecto de las perturbaciones sobre la riqueza y composición teniendo en cuenta tres factores usualmente relacionados con los cambios en diversidad: cobertura de dosel arbóreo (presencia - ausencia), tipo de vegetación (nativa - exótica) y ganado (presencia - ausencia). Frente a estos factores, se esperaba un gradiente de respuesta en riqueza y composición de la comunidad de coleópteros copro-necrófagos del bosque nativo, desde aquellos usos de la tierra que conservan la cobertura de dosel arbóreo y la vegetación nativa hasta aquellos que pierden la cobertura de dosel arbóreo y presentan vegetación exótica. A partir de estos estudios, el objetivo principal de este trabajo es generar recomendaciones de manejo de los ambientes disturbados que permitan conservar la diversidad de coleópteros copro-necrófagos y sus interacciones.

\section{METODOLOGÍA}

\section{Área de estudio}

Los muestreos fueron realizados en el Bosque Atlántico de Argentina, bosque semideciduo con un promedio de precipitaciones de $2000 \mathrm{~mm}$ distribuidos a lo largo de todo el ańo y un promedio de temperatura de $25^{\circ} \mathrm{C}$ en verano y $15{ }^{\circ} \mathrm{C}$ en invierno (Oliveira-Filho y Fontes, 2000). El paisaje queda definido por grandes extensiones de bosque nativo continuo en áreas protegidas (Parque Nacio- nal Iguazú, Parque Provincial Urugua-í, Parque Provincial Península, Reserva privada de Fundación Vida Silvestre, entre otras), plantaciones de árboles exóticos (principalmente Pinus taeda Linnaeus 1753) con y sin ganado, ambientes abiertos con ganado y pastos exóticos, cultivos anuales en pequeña escala (tales como tabaco y maíz) y plantaciones de yerba mate (Ilex paraguariensis Saint-Hilaire 1822) (Izquierdo et al., 2008; Zurita y Bellocq, 2012). Los coleópteros copro-necrófagos fueron muestreados durante la primavera (noviembre-diciembre) de 2014 y 2016, época del año con mayor actividad de estos coleópteros (Hernández y Vaz-de-Mello, 2009; da Silva et al., 2013).

Para llevar a cabo estos muestreos fueron seleccionados cuatro usos de la tierra (Figura 1) (cinco réplicas por uso de tierra) que difieren en los tres factores mencionado en la introducción: presencia/ausencia de dosel arbóreo, vegetación nativa o exótica y presencia/ausencia de ganado. Así, los ambientes muestreados fueron (A) plantaciones maduras de pinos (10-12 de edad) (Pinus taeda) ("plantaciones de pinos"; cobertura de dosel exótica sin ganado); (B) bosque nativo con ganado ("parquizados"; cobertura de dosel nativa con ganado); (C) plantaciones maduras de pinos (Pinus taeda) con ganado ("silvopastoriles"; cobertura de dosel exótica con ganado) y (D) áreas deforestadas de pastura con ganado ("potreros para ganadería"; sin cobertura de dosel y con ganado). Adicionalmente, cinco réplicas de bosque nativo continuo fueron muestreadas en áreas protegidas (Figura 1). La descripción detallada de cada uno de los ambientes se presenta en Tabla MS1. La distancia entre réplicas (25 en total) fue de al menos $1 \mathrm{~km}$ para garantizar independencia entre ellas (da Silva y Hernández, 2015). Para incrementar la representatividad regional, las réplicas fueron ubicadas en cuatro áreas separadas por una distancia mínima de $30 \mathrm{~km}$. Todos los usos de la tierra, incluido el bosque nativo, estuvieron representados en todas las áreas. 


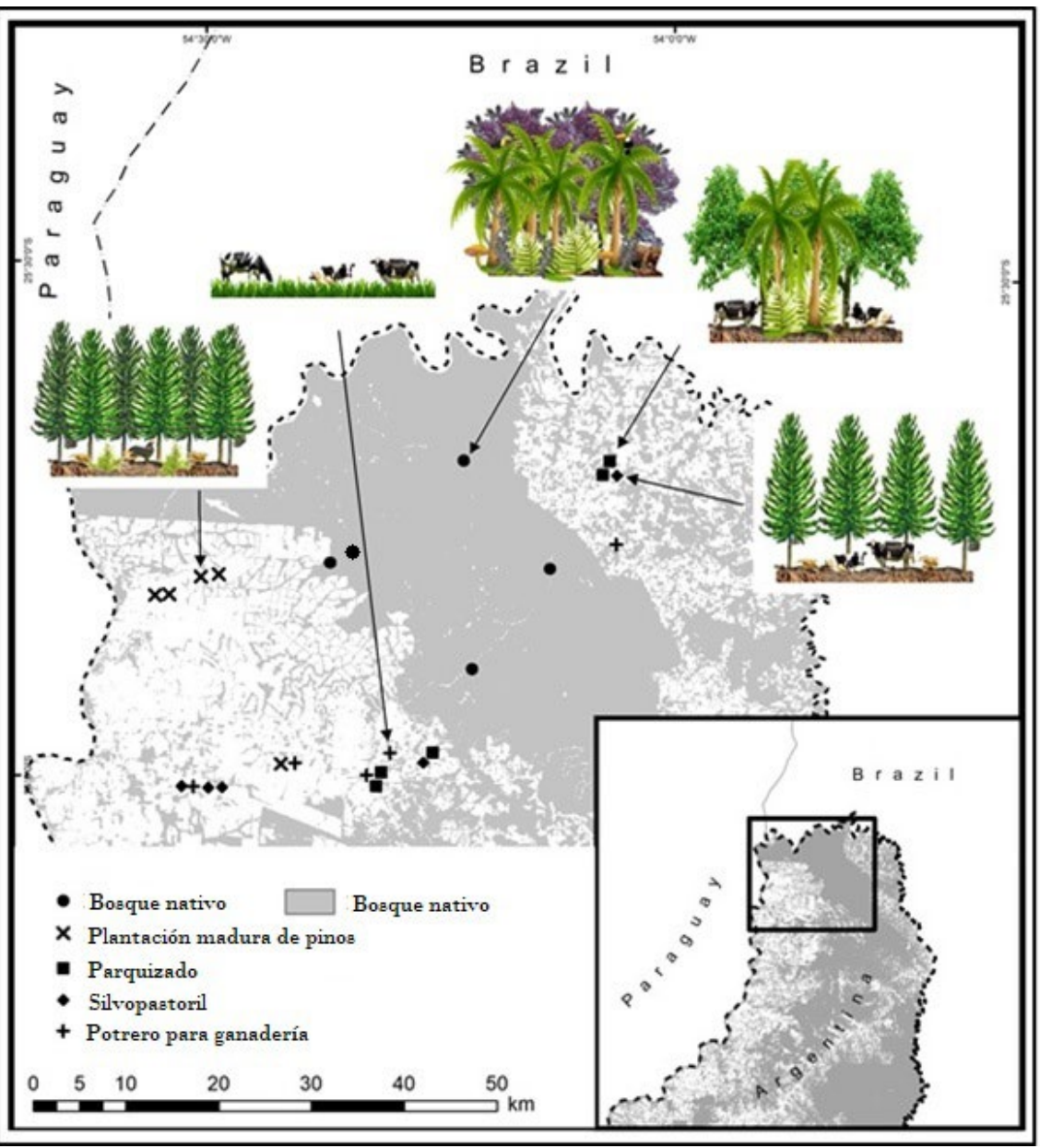

Figura 1. Bosque Atlántico de Argentina, con los diferentes usos de la tierra. Círculos: bosque nativo, cruces: plantación madura de pinos, cuadrados: parquizado, rombos: silvopastoril y signos positivos: potrero para ganadería. Modificado de Giménez Gómez et al., 2018a.

\section{Muestreo de coleópteros copro-necrófagos}

En cada una de las réplicas (25 en total, cinco de bosque nativo y cinco de cada uso de la tierra) se estableció una trama de $150 \mathrm{~m}$ x $150 \mathrm{~m}$. En cada trama, se colocaron 16 trampas de caída separadas por $50 \mathrm{~m}$ para minimizar la interferencia entre ellas (16 trampas $\mathrm{x} 25$ sitios $=400$ trampas) (Larsen y Forsyth, 2005; Tshikae et al., 2013). En total se realizaron cuatro períodos de muestreo, cada uno de 72 hs (12 días en total). En cada período se colectó el material y se renovó el cebo de cada trampa. Todas las muestras fueron preservadas en alcohol $70 \%$ hasta su posterior procesamiento. Los individuos colectados fueron depositados en la Colección Scarabaeidae del Instituto de Biología Subtropical- Iguazú (IBSI Sca), Misiones, Argentina.

Este muestreo fue repetido dos veces, en el 2014 para evaluar el efecto de la alteración del medio sobre la preferencia trófica de los coleópteros copronecrófagos ("estudio del nicho trófico") y en el 2016 para evaluar el efecto de los factores propuestos (cobertura de dosel arbóreo, ganado y tipo de vegetación) sobre la riqueza y composición ("estudio de diversidad"). La diferencia entre ambos muestreos fue los cebos utilizados.

Para el muestreo de nicho trófico, las 16 trampas por réplica fueron cebadas al azar con siete cebos, que se conocía a priori que los coleópteros copronecrófagos utilizan en el ambiente natural, y dos trampas no fueron cebadas como control. De las 16 trampas, se cebaron dos con cada uno de los siguientes recursos potenciales: 1) estiércol de felino (Leopardus pardalis Linnaeus 1758), 2) estiércol de corzuela (Mazama nana Hensel 1872), 3) estiércol de tapir (Tapirus terrestris Linnaeus 1758), 4) estiércol de mono (Alouatta caraya Humboldt 1812 y Sapajus cay Illiger 1815), 5) frutos en descomposición (Syagrus romanzoffiana Grassman 1968 y Chrysophyllum gonocarpum Mart y Eichler 1890), 6) carroña de vertebrados (pollo en descomposición) 
y 7) hongos en descomposición (Ascomycetes y Basidiomycetes) (Figura 2). Los cebos de carroña fueron extraídos del refrigerador 12 horas antes de ser colocados en la trampa y el proceso de descomposición final ocurrió en el campo durante las 72 horas de cada periodo de muestreo. El estiércol de felino, de corzuela y de mono fue obtenido en un refugio de animales salvajes ("Guira Oga"); mientras que el estiércol de tapir, los frutos y hongos fueron colectados en campo en los sitios de estudio.

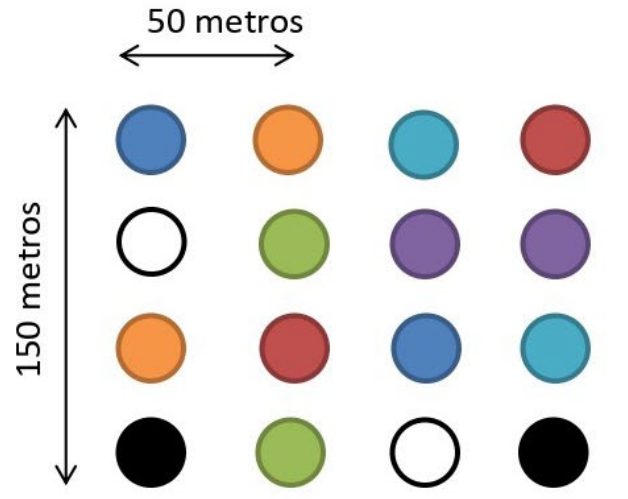

Para el muestreo de diversidad, de las 16 trampas ocho fueron cebadas con estiércol de humano y ocho con carroña de vertebrado (Figura 3); estos cebos seleccionados son los más utilizados para capturar especies de coleópteros coprófagas y necrófagas (Halffter y Mattherws, 1966; Spector, 2006).

Figura 2. Diseño de muestreo para muestreo de nicho trófico. El diseño consistió en una trama de $150 \mathrm{~m}$ x $150 \mathrm{~m}$ en la cual se colocaron 16 trampas de caída separas entre sí por $50 \mathrm{~m}$. Las trampas fueron cebadas con siete tipos de recursos que los coleópteros copro-necrófagos consumen en su ambiente natural (bosque nativo) y están representadas en esta figura por el color de los círculos: Círculo azul = carroña, rojo = estiércol de felino, verde = estiércol de tapir, violeta $=$ estiércol de corzuela, naranja $=$ hongos en descomposición, celeste = frutos en descomposición, negro = estiércol de mono y los círculos vacíos representan el control, es decir trampas que no fueron cebadas. En cada una de las réplicas la distribución de los cebos fue totalmente al azar.

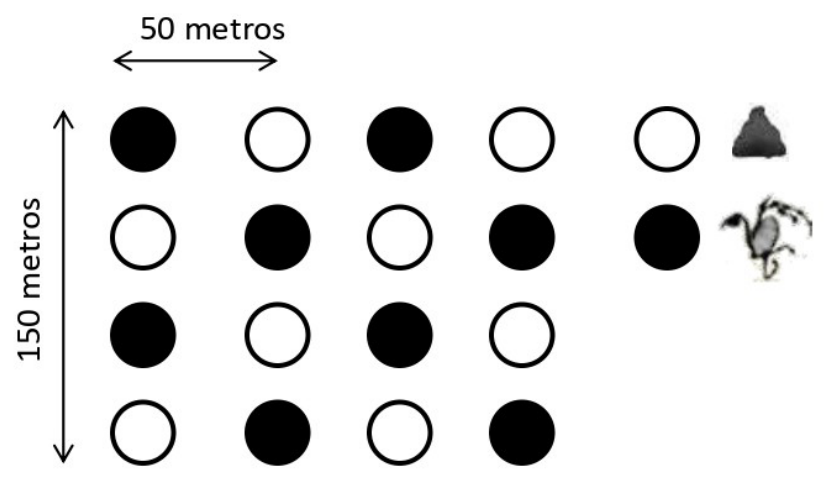

Figura 3. Diseńo de muestreo para caracterizar la diversidad en el Bosque Atlántico de Argentina. El diseńo consistió en una trama de $150 \mathrm{~m} \times 150 \mathrm{~m}$ en la cual se colocaron 16 trampas de caída separas entre sí por $50 \mathrm{~m}$. Las trampas fueron cebadas con dos tipos de cebos principales que los coleópteros copro-necrófagos consumen y están representadas en esta figura por el color de los círculos, círculo negro = carrońa y círculo blanco = estiércol humano. 


\section{Análisis de datos}

En ambos muestreos la cobertura de muestreo fue estimada a partir del software libre INEXT (Chao et al., 2016). A nivel de comunidad, la riqueza de especies entre ambientes fue comparada a través de un análisis no paramétrico, Kruskal-Wallis, y análisis a posteriori utilizando el paquete de $\mathrm{R}$ 'conover. prueba' (Dinno, 2017; R Core Team 2017).

Para explorar la selección de recursos de cada una de las especies en cada uno de los ambientes (diferentes usos de la tierra y bosque nativo), a partir del muestreo de nicho trófico (2014), se realizó un análisis factorial de correspondencia (FCA) por ambiente utilizando la frecuencia relativa de captura de cada especie en cada cebo (PAST 2.16; Hammer et al., 2001).

Para evaluar las diferencias en composición de especies entre los diferentes usos de la tierra y el bosque nativo, a partir del muestreo de diversidad (2016), se realizó un análisis no paramétrico multidimensional (NMDS) utilizando la transformación de logaritmo, Log $(x+1)$, y el índice de Bray-Curtis (Clarke, 1993). Las diferencias estadísticas entre los grupos formados a partir del NMDS fueron comparados con un análisis permutacional multivariado no paramétrico (PERMANOVA). El valor de $p$ fue calculado luego de 999 permutaciones. Tanto el NMDS como el PERMANOVA fueron realizados con el software PRIMER 6 + Permanova (Clarke y Gorley, 2006).

Finalmente, para explorar la influencia de la cobertura de dosel arbóreo (presencia-ausencia), tipo de vegetación (nativa-exótica) y ganado (presenciaausencia) en la composición de los ensambles de coleópteros copro-necrófagos de los diferentes usos de la tierra y el bosque nativo, se realizó otro PERMANOVA pero utilizando la función Adonis del paquete 'vegan' de R (Oksanen et al., 2017; R Core Team 2017). Las réplicas fueron utilizadas para realizar el PERMANOVA y en el modelo fueron considerados los factores (cobertura de dosel arbóreo, tipo de vegetación y ganado) como variables explicatorias. La función Adonis proporciona como resultado el valor de su estadístico $(F)$, una medida del tamaño del efecto (R2) y el valor de $p(p)$ para cada factor. Los factores con un $p<0.05$ se consideran significativos y mientras mayor sea el valor de R2 mayor es el poder explicativo del factor.

\section{RESULTADOS}

\section{Estudio del nicho trófico}

A partir del muestreo de campo se colectaron 7.393 individuos correspondientes a 44 especies, 26 de las cuáles fueron capturadas en el bosque nativo, 25 en la plantación de pino, 18 en parquizado, 17 en silvopastoril y 11 en potrero para ganadería (Tabla MS2, Giménez Gómez et al., 2018a). La representatividad del muestreo fue mayor al $95 \%$ en todos los ambientes, por lo que se considera que fue completo. En relación a la comparación de riqueza, se encontraron diferencias significativas entre ambientes $(\mathrm{K}-\mathrm{W}$, $H=16.84, n=5, p=0.0019)$, siendo la misma mayor en ambientes que conservan la cobertura de dosel arbóreo y menor en el ambiente sin cobertura de dosel arbóreo (Figura 4).

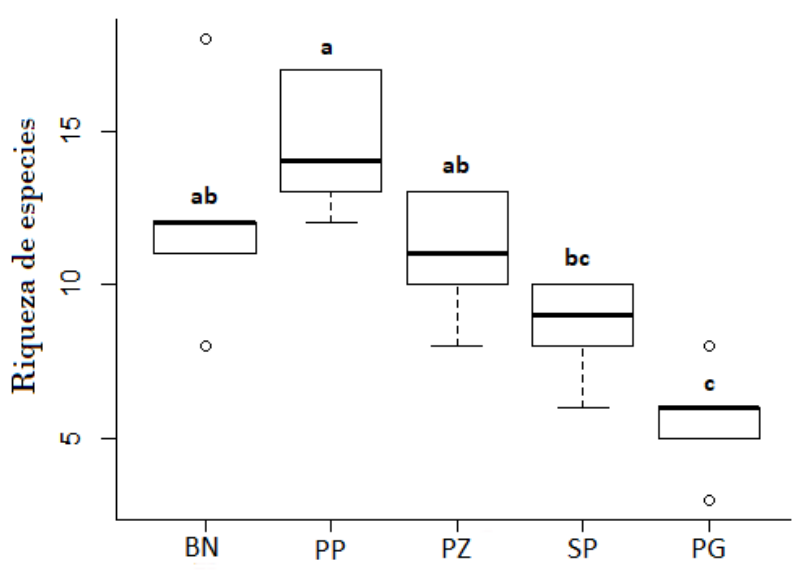

Figura 4. Riqueza de especies de coleópteros copro-necrófagos (cuartiles, mediana y valores atípicos) en el bosque nativo $(\mathrm{BN})$ y cuatro usos de la tierra $(\mathrm{PP}=$ plantación de pino, $\mathrm{PZ}=$ parquizado, $\mathrm{SP}=$ silvopastoril y $\mathrm{PG}=$ potrero para ganadería) en el Bosque Atlántico de Argentina. En este caso, para estimar los cambios en diversidad fueron utilizados siete tipos de cebos (estiércol de mono, felino, corzuela y tapir, carroña de vertebrados, frutos y hongos en descomposición) y el blanco. Medianas con una letra en común no son significativamente diferentes ( $p>0.05)$. Modificado de Giménez Gómez et al., 2018a. 
A partir de los FCAs se observan asociaciones específicas entre las especies de coleópteros copronecrófagos y los cebos en cada uno de los ambientes (Figura 5). En todos los ambientes se observa que las especies coprófagas del género Dichotomius Hope 1838, Eurysternus Dalman 1824 y algunas especies del género Canthon Hoffmannsegg 1817, presentan mayor preferencia por el estiércol de mono y felino que sobre el de tapir y venado, mientras que las espe- cies necrófagas del género Deltochilum Eschscholtz 1822, Coprophanaeus D'Olsoufieff 1924 y algunas especies del género Canthon, presentan similar preferencia por la carrońa y hongos en descomposición y casi no fueron atraídas por las frutas en descomposición. Sólo en el bosque nativo, algunas especies como Dichotomius sericeus Harold 1867, Uroxys sp. 1 y Eurysternus caribaeus Herbs 1789, presentan una atracción por los frutos en descomposición.
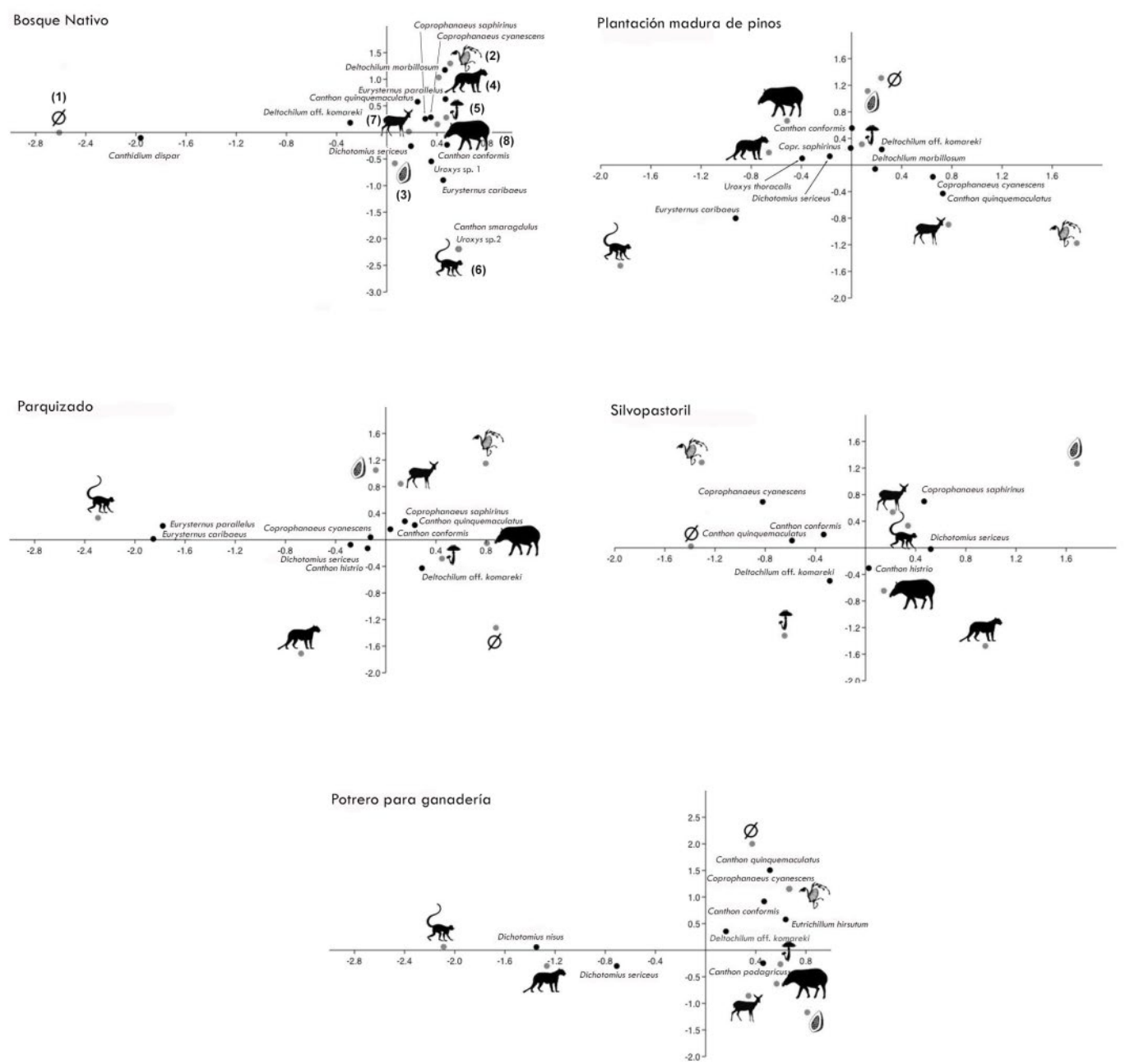

Figura 5. Análisis factorial de correspondencia basado en la selección de recursos de los coleópteros copro-necrófagos en el bosque nativo y cuatro usos de la tierra (plantación de pino, parquizado, silvopastoril y potrero para ganadería) en el Bosque Atlántico de Argentina. Los números en la primer figura (bosque nativo) corresponden a los cebos utilizados: (1) control, (2) carrońa, (3) frutos en descomposición, (4) estiércol de felino, (5) hongos en descomposición, (6) estiércol de mono, (7) estiércol de corzuela y (8) estiércol de tapir. Modificado de Giménez Gómez et al., 2018a. 


\section{Estudio de diversidad}

A partir del muestreo de campo, fueron colectados 14.712 individuos correspondientes a 47 especies de 16 géneros de la subfamilia Scarabaeinae. De las 47 especies, 27 fueron capturadas en bosque nativo, 24 en la plantación de pino, 31 en el parquizado, 24 en el silvopastoril y 19 en potrero para ganadería (Tabla MS3, Giménez Gómez et al., 2018b).

Respecto a la cobertura de muestreo, todos los ambientes presentaron una cobertura mayor al 98 $\%$. Cuando se compara la riqueza de especies, se encontraron diferencias significativas entre ambientes (K-W, $H=11.30, n=4, p=0.0212)$. Similar a lo observado en el muestreo de nicho trófico, todos los ambientes que conservan la cobertura de dosel arbóreo (bosque nativo, plantación de pino, parquizado y silvopastoril) presentaron similar y mayor riqueza de especies que el ambiente que pierde la cobertura de dosel arbóreo (potrero para ganadería) (Figura 6).

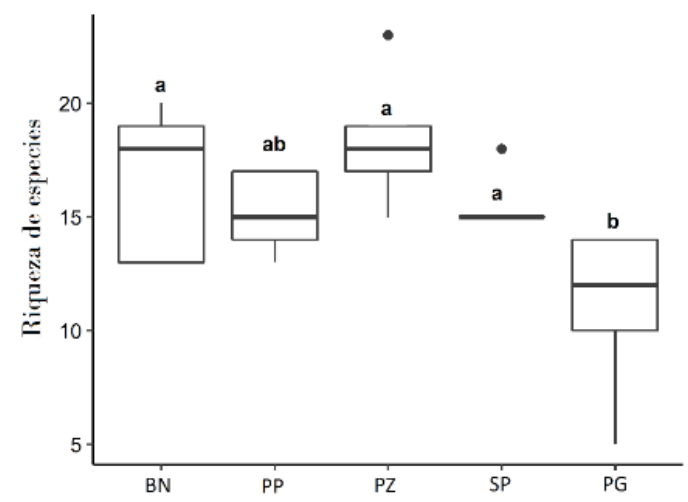

Figura 6. Riqueza de especies de coleópteros copronecrófagos (cuartiles, mediana y valores atípicos) en el bosque nativo $(\mathrm{BN})$ y cuatro usos de la tierra $(\mathrm{PP}=$ plantación de pino, $\mathrm{PZ}=$ parquizado, $\mathrm{SP}=$ silvopastoril y $\mathrm{PG}=$ potrero para ganadería) en el Bosque Atlántico de Argentina. En este caso, para estimar los cambios en diversidad, fueron utilizados dos tipos de cebos: carroña de vertebrados y estiércol humano. Medianas con una letra en común no son significativamente diferentes ( $\mathrm{p}$ $>0.05$ ). Modificado de Giménez Gómez et al., 2018b.
Cuando se compara la composición de especies entre los diferentes usos de la tierra y el bosque nativo, el NMDS separa el potrero para ganadería del bosque nativo y del resto de usos de la tierra, que conservan la cobertura de dosel arbóreo (Figura 7). También el PERMANOVA muestra que hay diferencias significativas entre los ambientes (PERMANOVA, $F=12.348, d f=4, p=0.001$ ), con la única excepción del parquizado con el silvopastoril (Tabla MS4). Finalmente, el PERMANOVA realizado a través de la función Adonis muestra que los tres factores propuestos (cobertura de dosel arbóreo, tipo de vegetación y ganado) explican las diferencias en composición encontradas entre ambientes pero en diferente magnitud: la cobertura de dosel arbóreo es el factor con mayor influencia $(p=0.001, \mathrm{R} 2=$ $0.541)$, luego el ganado $(p=0.002, \mathrm{R} 2=0.094) \mathrm{y}$ por último el tipo de vegetación $(p=0.046, \mathrm{R} 2=$ 0.043 ) (Tabla 1).

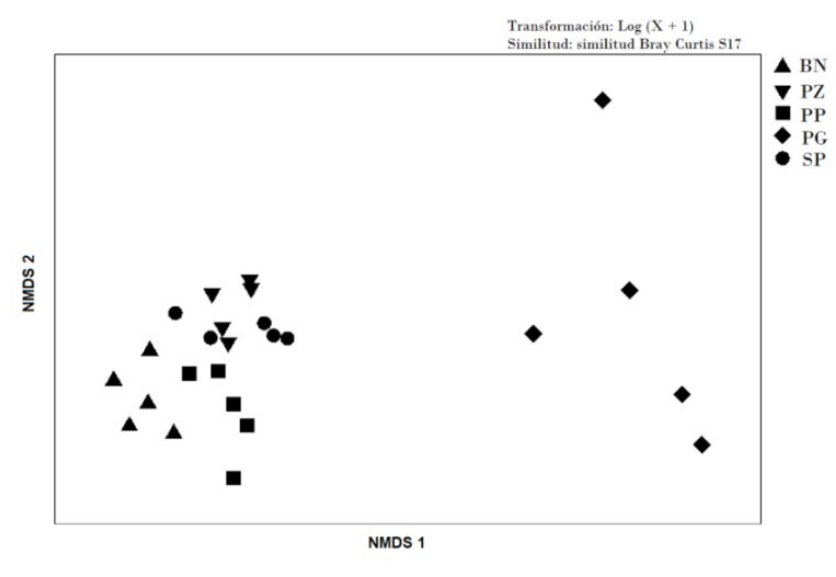

Figura 7. Análisis no paramétrico multidimensional (NMDS) basado en la composición de especies de la comunidad de coleópteros copro-necrófagos en el bosque nativo y cuatro usos de la tierra en el bosque Atlántico de Argentina. Triángulos = bosque nativo $(\mathrm{BN})$, cuadrados $=$ plantación de pino $(\mathrm{PP})$, triángulos invertidos $=$ parquizado (PZ), puntos = silvopastoril $(\mathrm{SP})$ y diamantes = potrero para ganadería $(\mathrm{PG})$. Modificado de Giménez Gómez et al., 2018b. 
Tabla 1. Resultados del PERMANOVA (a través de la función 'Adonis'de R, utilizando el índice de Bray Curtis como medida de disimilitud y 999 permutaciones) entre tres factores: cobertura de dosel arbóreo (presencia-ausencia), tipos de vegetación (nativa-exótica) y ganado (presencia-ausencia), para evaluar la influencia esos tres factores en la composición de especies de los coleópteros copro-necrófagos en el bosque nativo y cuatro usos de la tierra (plantación de pino, parquizado, silvopastoril y potrero para ganadería) en el Bosque Atlántico de Argentina. Gl = grados de libertad, $\mathrm{SC}$ = suma de cuadrados, $\mathrm{CM}$ = cuadrado medio, $\mathrm{F}=$ estadístico, $\mathrm{R} 2$ = "tamańo del efecto", $\mathrm{p}$ $=\mathrm{p}$ valor. ${ }^{* * *} \mathrm{p} \leq 0.001,{ }^{* *} \mathrm{p}<0.01,{ }^{*} \mathrm{p}<0.05$. Mayor $\mathrm{R} 2$ indica mayor poder explicativo. Modificado de Giménez Gómez et al., 2018b.

\begin{tabular}{c|c|c|c|c|c|c} 
FACTORES & Gl & SC & $\mathbf{C M}$ & $\boldsymbol{F}$ & $\mathbf{R}^{\mathbf{2}}$ & $\boldsymbol{p}$ \\
\hline Cobertura de dosel arbóreo (presencia-ausencia) & 1 & 2.210 & 2.210 & 35.283 & $\mathbf{0 . 5 4 1}$ & $\mathbf{0 . 0 0 1}^{* * *}$ \\
\hline Tipo de vegetación (nativa-exótica) & 1 & 0.176 & 0.177 & 2.804 & $\mathbf{0 . 0 4 3}$ & $\mathbf{0 . 0 4 6}^{*}$ \\
\hline Ganado (presencia-ausencia) & 1 & 0.384 & 0.384 & 6.134 & $\mathbf{0 . 0 9 4}$ & $\mathbf{0 . 0 0 2}^{* *}$ \\
\hline Residuos & 21 & 1.315 & 0.063 & & $\mathbf{0 . 3 2 2}$ &
\end{tabular}

\section{DISCUSIÓN}

En los estudios considerados en este trabajo se esperaba que aquellos usos de la tierra que preservan la cobertura de dosel arbóreo y la vegetación nativa conservaran también la diversidad (riqueza y composición) de coleópteros copro-necrófagos con respecto al bosque nativo. Los resultados obtenidos apoyan parcialmente lo esperado, ya que tanto la riqueza como la composición de especies tienden a ser similares a las del bosque nativo en aquellos ambientes que conservan la cobertura de dosel arbóreo con vegetación nativa (parquizado) o exótica (plantación de pino y silvopastoril), y es diferente en ambientes sin cobertura de dosel arbóreo (potrero para ganadería). De los tres factores estudiados, la presencia de cobertura de dosel arbóreo es el factor que más influye en la conservación de las comunidades de coleópteros copro-necrófagos en los ambientes estudiados, el ganado y el tipo de vegetación también influyen pero en menor medida. Estudios previos, llevados a cabo en el Bosque Atlántico, han explorado la influencia de la cobertura de dosel arbóreo, condiciones microclimáticas y presencia de ganado en respuesta a las perturbaciones de origen antrópico (Hernández et al., 2014; da Silva y Hernández, 2016; Bogoni et al., 2014; Gómez-Cifuentes et al., 2017); sin embargo, hasta el momento no se habían realizado estudios que pusieran a prueba todos los factores juntos, ni tampoco estudios que evaluaran si los cambios en diversidad encontrados se encuentran asociados a algún componente o algunos componentes del nicho de las especies de coleópteros copro-necrófagos.

Un gran número de estudios han mostrado que el reemplazo, la fragmentación y la degradación de los bosques tropicales y subtropicales cambian la abundancia (Sánchez-de-Jesús et al., 2016), riqueza y composición de especies (Halffter y Arellano, 2002; Scheffler, 2005; Nichols et al., 2007; Gardner et al., 2008; Neita y Escobar, 2012; Peyras et al., 2012; Audino et al., 2014; Hernández et al., 2014; Gómez-Cifuentes et al., 2017). En general, la magnitud de esos cambios ha sido asociada con la pérdida de recursos tróficos, como estimación indirecta a partir de la pérdida de grandes mamíferos, y con los cambios en las condiciones ambientales (Nichols et al., 2007; Hernández et al., 2014; Hewavithana et al., 2016; Gómez-Cifuentes et al., 2017). Los resultados de este trabajo concuerdan con los obtenidos por Alvarado et al. (2018) en los cuales se obtuvo también que la pérdida de cobertura de dosel arbóreo es el factor que más influye en los cambios de diversidad, seguido por la presencia de ganado. La pérdida de cobertura de dosel arbóreo tiene un efecto indirecto en los coleópteros copro-necrófagos dado que se encuentra asociada directamente con cambios que se producen en las condiciones microclimáticas como la radiación solar, la temperatura y humedad ambiental, la intensidad 
de luz y la temperatura y humedad del suelo (Halffter et al., 1992; Davis et al., 2002; Tuff et al., 2016). Los coleópteros copro-necrófagos son sensibles a los cambios en las condiciones microclimáticas (Davis et al., 2000; Duncan y Byrne, 2000; Chown, 2001; Nichols et al., 2007), por lo tanto, cambios en éstas los afectan directamente.

Con respecto a la presencia de ganado, su efecto secundario puede estar asociado a que, si bien las especies de coleópteros copro-necrófagos son principalmente coprófagas, el estiércol de vaca constituye un recurso de baja calidad (bajo nivel nutritivo) (Fincher et al., 1970; Filgueiras et al., 2009; Marsh et al., 2013). Para el grupo de estudio ya ha sido demostrado que las especies coprófagas presentan preferencias por el estiércol de omnívoro sobre el de carnívoro o herbívoro, sobre todo porque el de omnívoro les aporta más nutrientes (Fincher et al., 1970; Cambefort y Hanski, 1991; Filgueiras et al., 2009; Marsh et al., 2013). Esto concuerda con los resultados obtenidos a partir de los FCAs, en los cuáles se observa que en los ambientes con ganado (parquizado, silvopastoril y potrero para ganadería) las especies coprófagas prefieren estiércol de omnívoro (mono) o carnívoro (felino) sobre el de herbívoro (corzuela) o frugívoro (tapir). En este trabajo se esperaba que, en ambientes con ganado, las especies coprófagas prefirieran estiércol similar al de las vacas (estiércol de tapir por ejemplo), pero los resultados no fueron los esperados. Esto sugiere que podría existir un fuerte componente filogenético en la selección de recursos por parte de estas especies. Por otro lado, el hecho de que las especies necrófagas prefieran por igual carroña de vertebrados y hongos en descomposición podría estar asociado a que ambos recursos comparten componentes y organismos que participan en su descomposición y que son la principal fuente de recursos para este grupo (Anduaga y Halffter, 1993; Bustos-Gómez y Lopera, 2003; Schmitte et al., 2004). Finalmente, el hecho de que la riqueza y composición de especies se reduzca en ambientes abiertos, a pesar de que la oferta de recursos es la misma en todos los ambientes, sugiere que si bien la preferencia de recursos de las especies es importante y se ve afectada por las alteraciones del medio, no sería el principal limitante a la hora de hacer uso de un ambiente perturbado. Otros componentes del nicho, como la tolerancia térmica por ejemplo, probablemente tengan un efecto mayor.

Finalmente, la menor influencia del tipo de vegetación en la diversidad de especies de coleópteros copro-necrófagos no es sorprendente dado que muy pocas especies de estos coleópteros utilizan material vegetal para alimentarse y nidificar (Halffter y Matthews, 1966; Monteith y Storey, 1981; Scholtz et al., 2004; Davis et al., 2008; Halffter y Halffter 2009; Holter et al., 2009). Dentro del ensamble de coleópteros copro-necrófagos del bosque Atlántico de Argentina, sólo una especie, Dichotomius carbonarius Mannerheim 1829, ha sido observada utilizando material vegetal para nidificar y alimentarse (Dinghi et al., 2013). El estudio de la influencia del tipo de vegetación en la diversidad de coleópteros copro-necrófagos no había sido puesto a prueba hasta el momento; así, a pesar de su baja influencia, los resultados obtenidos para este factor resultan novedosos para el grupo de estudio.

\section{CONCLUSIÓN}

Los resultados de estos estudios y de estudios previos sugieren que las condiciones microclimáticas del sotobosque (afectadas por la presencia/ausencia de cobertura de dosel arbóreo), más que la presencia de ganado o el tipo de vegetación, determinan las comunidades de coleópteros copro-necrófagos en diferentes usos de la tierra en regiones tropicales y subtropicales. Estos resultados permiten generar una simple pero poderosa recomendación para el manejo de ambientes perturbados: la conservación de la cobertura de dosel arbóreo, sobre todo si esa cobertura es nativa, es fundamental para conservar la comunidad de coleópteros copro-necrófagos y, junto con ella, las funciones que éstos ejercen sobre el ecosistema. De este modo, por ejemplo, al conservar la cobertura de dosel arbóreo y por lo tanto la diversidad de coleópteros copro-necrófagos, se conservaría una de las funciones ecosistémicas más importantes llevadas a cabo por ellos, el enterramiento de materia orgánica. Esta función, a su vez, ayudaría a mantener 
el ciclo de nutrientes y la bioturbación del suelo que permiten a las plantas utilizar los recursos del suelo eficientemente (Bornemissza, 1970; Galbiati et al., 1995; Bang et al., 2005; Hanafy, 2012), así como también reducir la depredación de semillas (Estrada y Coates-Estrada, 1991), ayudando de este modo al crecimiento, reproducción y supervivencia de las plantas (Figura 8). Si bien esta recomendación de manejo había sido sugerida en trabajos previos (Nichols et al., 2007; Alvarado et al., 2018), los resultados obtenidos en este estudio han puesto claramente de manifiesto la gran importancia de la cobertura de dosel arbóreo en la conservación de las comunidades de coleópteros copro-necrófagos.

\section{AGRADECIMIENTOS}

Queremos agradecer a Fernando Foletto, Andrés Gómez-Cifuentes, Ana Munevar, Gisele Jaime, Natalia Vespa, Soledad Soto, Agustín Solari y Mariano Giombini por ayudarnos con el trabajo de campo asociado a los estudios presentados. A Fernando Vaz-de-Mello por ayudarnos con la identificación de las especies, a Santiago Velazco por ayudarnos con los análisis y Ariel Insaurralde por su ayuda con el mapa. Agradecemos también la hospitalidad y buena predisposición de los dueños de las chacras y de los guardaparques, y a los dueńos y personal de Güira Oga por ayudarnos con la

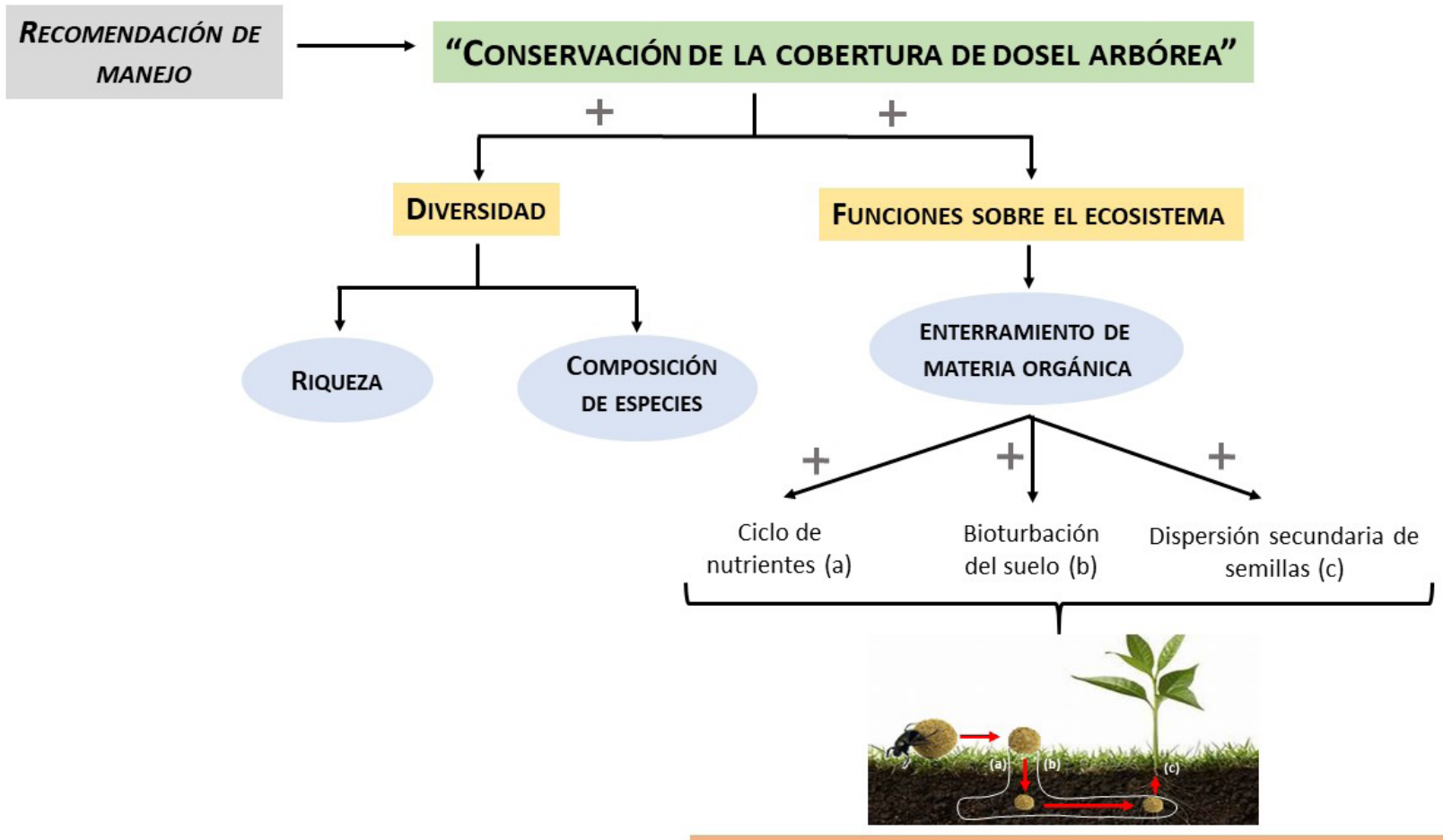

CRECIMIENTO, REPRODUCCIÓN Y SUPERVIVENCIA DE LAS PLANTAS

Figura 8. Recomendación de manejo de ambientes perturbados que permitiría la conservación de la diversidad de coleópteros copro-necrófagos en el Bosque Atlántico de Argentina y las funciones que llevan a cabos en el ecosistema, dentro de las que se destaca el enterramiento de materia orgánica. Esta función no sólo es importante porque estimula positivamente al ciclo de nutrientes (a) y bioturbación del suelo (b), sino además porque permite la dispersión de semillas (c) y su protección frente a los depredadores. Todo ello conlleva finalmente a un mayor crecimiento, reproducción y supervivencias de las plantas. 
colecta de cebos. Los financiamientos utilizados para llevar a cabo los estudios presentados en este trabajos son los siguientes: CONICET (Proyecto UE IBS \# 22920160100130CO otorgado a Mario Di Bitetti), UCAR-MAGyP (BIO 23, PIA 10105-14057 otorgados a Gustavo Zurita) y ANPCyT (PICT-PRH 2702 otorgado a Gustavo Zurita). Finalmente, los permisos de colecta de coleópteros copro-necrófagos fueron otorgados por la Administración de Parques Nacionales, el Ministerio de Ecología y Arauco Argentina S.A.

\section{REFERENCIAS}

Alvarado, F., Escobar, F., Williams, D.R., Arroyo-Rodriguez, V. y Escobar-Hernández, F. (2018). The role of livestock intensification and landscape structure in maintaining tropical biodiversity. Journal of Applied Ecology, 55:185194. https://doi.org/10.1111/1365-2664.12957.

Andrade-Núñez, M.J. y Aide, T.M. (2010). Effects of habitat and landscape characteristics on medium and large mammal species richness and composition in northern Uruguay. Zoologia, 27: 909-917. http://www. scielo.br/pdf/zool/v27n6/12.pdf.

Andresen, E. (2003). Effect of forest fragmentation on dung beetles communities and functional consequences for plant regeneration. Ecography, 26: 87-97. https://doi. org/10.1034/j.1600-0587.2003.03362.x.

Andresen, E. y Feer, F. (2005). The role of dung beetles as secondary seed dispersers and their effect on plant regeneration in tropical rainforests. En: Forget, P.M., Lambert, J.E., Hulme, P.E. y Vander Wall, S.B. (eds.). Predation, Dispersal and Seedling Establishment. CABI International, Wallingford, UK. pp 331-349.

Anduaga, S. y Halffter, G. (1993). Nidificación y alimentación en Lia tongus rhinocerulus (Bates) (Coleoptera:Scarabaeidae: Scarabaeinae). Acta Zoologica Mexicana, 57: 1-4. http://www.acuedi.org/doc/2833/ nidificacin-y-alimentacin-en-lia-tongus-rhinocerulus$\% 28$ bates \% 29-\%28coleoptera-scarabaeidaescarabaeinae $\% 29 . \mathrm{html}$.

Arellano, L., León-Cortés, J. y Halffter, G. (2008). Response of dung beetle assemblages to landscape structure in remnant natural and modified habitats in southern Mexico. Insect Conservation and Diversity, 1: 253-262. https://doi.org/10.1111/j.1752-4598.2008.00033.x.
Audino, I., Louzada, J. y Comita, L. (2014). Dung beetles as indicators of tropical forest restoration success: is it possible to recover species and functional diversity?. Biological Conservation, 169: 248-257. https:/doi. org/10.1016/j.biocon.2013.11.023.

Bang, H.S., Lee, J.H., Kwon, O.S., EunNa, Y., Jang, Y.S. y Kim, W.H. (2005). Effects of paracoprid dung beetles (Coleoptera: Scarabaeidae) on the growth of pasture herbage and on the underlying soil. Applied Soil Ecology, 29: 165-171. https://doi.org/10.1016/j. apsoil.2004.11.001.

Bogoni, J.A. y Hernández, M.I.M. (2014). Attractiveness of Native Mammal's feces of different trophic guilds to dung beetles (Coleoptera: Scarabaeinae). Journal of Insect Science, 14, 1, 1. https://doi.org/10.1093/jisesa/ ieu161.

Bornemissza, G.E. y Williams, C.H. (1970). An effect of dung beetles activity on plant yield. Pedobiologia, 10: 1-7. https://www.cabdirect.org/cabdirect/ abstract/19740516596.

Broennimann, O., Fitzpatrick, M.C., Pearman, P.B., Petitpierre, B., Pellissier, L., Yoccoz, N.G., Thuiller, W., Fortin, M.J., Randin, C., Zimmermann, N.E., Graham, C.H. y Antonie, G. (2012). Measuring ecological niche overlap from occurrence and spatial environmental data. Global Ecology and Biogeography, 21: 481-497. https:// doi.org/10.1111/j.1466-8238.2011.00698.x.

Bustos-Gómez, F. y Lopera Toro, A. (2003). Preferencia por cebo de los escarabajos coprofagos (Coleoptera: Scarabaeidae: Scarabaeinae) de un remante de bosque seco tropical al norte de Tolima (Colombia). Sociedad Entomológica Aragonesa, 30: 59-65.

Cabrera. (1971). Fitogeografía de Argentina. Boletín de sociedad Argentina de Botánica, Volúmen XIV, Nro 1-2.

Cambefort, Y. y Hanski, I. (1991). Dung beetle population biology. En: Hanski, I. y Camberfort, Y (eds.). Dung beetle ecology. Princeton University Press, Princeton, NJ. pp 36-50.

Campanello, P.I., Montti, L., Goldstein, G. y Mac Donagh, P. (2009). Reduced impact logging and postharvesting forest management in the Atlantic Forest: alternative approaches to enhance canopy tree growth and regeneration and to reduce the impact of invasive species. En: Grossberg, S.P. (ed.). Forest management. Nova Science. New York. pp 39-59. 
Chao, A., Ma, KH. y Hsieh, T.C. (2016). iNEXT (iNterpolation and EXTrapolation) Online: software for interpolation and extrapolation of species diversity. Program and user's guide. http://chao.stat.nthu.edu.tw/ wordpress/software_download/.

Chown, S.L. (2001). Physiological variation in insects: hierarchical levels and implications. Journal of Insect Physiology, 47:649-660. https://doi.org/10.1016/ S0022-1910(00)00163-3.

Christie, F.J. y Hochuli, D.F. (2008). Responses of wasp communities to urbanization: effects on community resilience and species diversity. Journal of Insect Conservation, 13: 213-221. https://link.springer.com/ article/10.1007/s10841-008-9146-5.

Clarke, K.R. (1993). Non-parametric multivariate analyses of changes in community structure. Austral Journal of Ecology, 18:117-143. https://doi. org/10.1111/j.1442-9993.1993.tb00438.x.

Clarke, K.R. y Gorley, R.N. (2006). PRIMER v6. User manual/tutorial. PRIMER-E, Plymouth.

Culot, L., Bovy, R., Vaz-de-Mello, F.Z., Guevara, R. y Galetti, M. (2013). Selective defaunation affects dung beetle communities in continuous Atlantic rainforest. Biological Conservation, 163: 79-89. https://doi. org/10.1016/j.biocon.2013.04.004.

Davies, K.F. y Margules, C.R. (1998). Effects of habitat fragmentation on carabid beetles: experimental evidence. Journal of Animal Ecology, 67: 460-471. https://doi. org/10.1046/j.1365-2656.1998.00210.x.

Davis, A.L.V. (1996). Seasonal dung beetle activity and dung dispersal in selected South African habitats: implications for pasture improvement in Australia. Agriculture, Ecosystems and Environment, 58: 157-169. https://doi.org/10.1016/0167-8809(96)01030-4.

Davis, A.L.V., Chown, S.L., McGeoch, M.A. y Scholtz, C.H. (2000). A comparative analysis of metabolic rate in six Scarabaeus species (Coleoptera: Scarabaeidae) from southern Africa: further caveats when inferring adaptation. Journal of Insect Physiology, 46:553-562. https://doi.org/10.1016/S0022-1910(99)00141-9.

Davis, A.L.V., Van Aarde, R.J., Scholtz, C.H. y Delport, J.H. (2002). Increasing representation of localized dung beetles across a chronosequence of regenerating vegetation and natural dune forest in South Africa. Global Ecology and Biogeography, 11: 191-209. https:// doi.org/10.1046/j.1466-822X.2002.00283.x.
Davis, A.L.V., Frolov, A.V. y Scholtz, C.H. (2008). The African dung beetle genera. Protea Book Publishers, Pretoria.

Da Silva, P.G., Vaz-de-Mello, F.Z. y Di Mare, R.A. (2013). Diversity and seasonality of Scarabaeinae (Coleoptera: Scarabaeidae) in forest fragments in Santa Maria, Rio Grande do Sul, Brazil. Annals of the Brazilian Academy of Sciences, 85: 679-697. http://www.scielo.br/pdf/ aabc/2013nahead/0001-3765-aabc-00-00-3313.pdf.

Da Silva, P.G. y Hernández, M.I.M. (2015). Spatial Patterns of Movement of Dung Beetle Species in a Tropical Forest Suggest a New Trap Spacing for Dung Beetle Biodiversity Studies. PLoS ONE, 10: e0126112. https:// doi.org/10.1371/journal.pone.0126112.

Da Silva, P.G. y Hernández, M.I.M. (2016). Spatial variation of dung beetle assemblages associated with forest structure in remnants of southern Brazilian Atlantic Forest. Revista Brasileira de Entomologia, 60: 73-81. https://doi.org/10.1016/j.rbe.2015.11.001.

De Angelo, C. (2009). El paisaje del bosque Atlántico del Alto Paraná y sus efectos sobre la distribución y estructura poblacional del jaguar (Panthera onca) y el puma (Puma concolor). Tesis doctoral. Universidad de Buenos Aires, Argentina. https://documentop.com/deangelo-2009-tesis-uba-el-paisaje-del-bosque-atlanticodel-alto-parana-y-sus-e_59fc9c3b1723dd376cb47113. html.

Dinghi, P.A., Sánchez, M.V., Cantil, L.F., Sarzetti, L.C. y Genise, J.F. (2013). Leaf-litter brood chambers in dichotomius (luederwaldtinia) carbonarius (mannerheim, 1829) (coleoptera: scarabaeidae): a novel behavior for dung beetles. Coleopterist Bulletin, 67:388396. https://doi.org/10.1649/0010-065X-67.3.388.

Dinno, A. (2017). Conover.prueba: Conover-Iman Prueba of Multiple Comparisons Using Rank Sums. $\mathrm{R}$ package version 1.1.4. https:/CRAN.R-project.org/ package $=$ conover. prueba.

Dirzo, R. y Raven, P.H. (2003). Global state of biodiversity and loss. Annual Reviews Environment and Resources, 28: 137-167. https://www.annualreviews.org/doi/ abs/10.1146/annurev.energy.28.050302.105532.

Di Bitetti, M.S., Placci, G. y Dietz, L.A. (2003). A biodiversity vision for the upper Paraná Atlantic Forest ecoregion: Designin a Biodiversity Conservation Landscape and Setting Priorities for Conservation Action. World Wild life Fund. Washington, D.C, U.S.A. https:/www.coursehero.com/file/29404962/ABiodiversity-Vision-for-the-Upper-Parana-AtlanticForest-Ecoregiondoc/. 
Duncan, F.D. y Byrne, M.J. (2000). Discontinuos gas exchange in dung beetles: patterns and ecological implications. Oecologia, 12: 452-458. https://link. springer.com/article/10.1007/s004420050966.

Estrada, A. y Coates-Estrada, R. (1991). Howler monkeys (Alouatta palliata), dung beetles (Scarabaeidae) and seed dispersal: ecological interactions in the tropical rain forest of Los Tuxtlas, Mexico. Journal of tropical ecology, 7: 459474. https://doi.org/10.1017/S026646740000585X.

Filgueiras, B.K.C., Liberal, C.N., Aguiar, C.D.M., Hernández, M.I.I. y Iannuzzi, L. (2009). Attractivity of omnivore carnivore and herbivore mammalian dung to Scarabaeinae (Coleoptera Scarabaeidae) in a tropical Atlantic Forest remnant. Revista Brasileira de Entomología, 53: 422-427. http://dx.doi.org/10.1590/ S0085-56262009000300017.

Fincher, G.T., Stewart, T.B. y Davis, R. (1970). Attraction of coprophagous beetles to dung of various animals. Journal of Parasitology, 56: 378-383. https://www.jstor. org/stable/3277680.

Galbiati, C., Bensi, C., Conceição, C.H.C., Florcoviski, J.F. y Calafiori, M.H. (1995). Estudo comparativo entre besouros do esterco Dichotomius anaglypticus (Mann., 1829) e Onthophagus gazella (F.), sobre a pastagem, em condiçóes brasileiras. Ecosistema, 20: 109-118.

Gardner, T.A., Hernández, M.I.M., Barlow, J. y Peres, C.A. (2008). Understanding the biodiversity consequences of habitat change: the value of secondary and plantation forests for neotropical dung beetles. Journal of applied ecology, 45: 883-893. https://doi.org/10.1111/j.13652664.2008.01454.x.

Giménez Gómez, V.C., Verdú, J.R., Gómez-Cifuentes, A., Vaz-de-Mello, F.Z. y Zurita, G.A. (2018a). Influence of land use on the trophic niche overlap of dung beetles in the semideciduous Atlantic forest of Argentina. Insect conservation and diversity, 11: 554-564. https://doi. org/10.1111/icad.12299.

Giménez Gómez, V.C., Verdú, J.R., Guerra Alonso, C. y Zurita, G.A. (2018b). Relationship between land uses and diversity of dung beetles (Coleoptera: Scarabaeinae) in the southern Atlantic forest of Argentina: which are the key factors?. Biodiversity and Conservation, 27: 32013213. https://doi.org/10.1007/s10531-018-1597-8.
Gómez-Cifuentes, A., Munevar, A., Gimenez, V.C., Gatti, M.G. y Zurita, G.A. (2017). Influence of land use on the taxonomic and functional diversity of dung beetles (Coleoptera: Scarabaeinae) in the southern Atlantic forest of Argentina. Journal of Insect Conservation, 21: 147-156. https://doi.org/10.1007/s10841-017-9964-4.

Gómez-Cifuentes, A., Giménez Gómez, V.C., Moreno, C.E. y Zurita, G.A. (2018). Dung beetles diversity in livestock areas in the southern Atlantic forest: the role of microclimatic conditions and soil structure. Basic and Applied Ecology, 34: 64-74. https://doi. org/10.1111/1365-2664.12957.

Halffter, G. y Matthews, E.G. (1966). The natural history of dung beetles of the subfamily Scarabaeinae (Coleoptera: Scarabaeidae). Sociedad Mexicana de Entomologia, 14: 1-312. https://doi.org/10.1002/mmnz.19690450211.

Halffter, G., Favila, M.E. y Halffter, V. (1992). A comparative study of the structure of the scarab guild in Mexican tropical rain forests and derived ecosystems. Revista Entomológica Mexicana, 84: 131-156.

Halffter, G. y Arellano, L. (2002). Response of Dung Beetle Diversity to Human-induced Changes in a Tropical Landscape. Biotropica, 34: 144-154. https://doi. org/10.1111/j.1744-7429.2002.tb00250.x.

Halffter, G. y Halffter, V. (2009). Why and where coprophagous beetles (Coleoptera: Scarabaeinae) eat seed, fruits or vegetable detritus. Boletín Sociedad Entomológica Aragonesa, 45: 1-22.

Hammer, O., Harper, D.A.T. y Ryan, P.D. (2001). Past: paleontological statistics software package for education and data analysis. Paleontología Electrónica, 4: 1-9.

Hanafy, H.E.M. (2012). Effect of dung beetles, Scarabaeus sacer (Scarabaeidae: Scarabaeinae) on certain biochemical contents of leaves and fruits of tomato and squash plants. Journal of Applied Science Research, 8: 4927-4936. http:// www.aensiweb.com/jasr/jasr/20133057734.

Hanski, I. y Cambefort, Y. (1991). Dung Beetle Ecology. Princeton University Press, Princeton.

Hernández, M.I.M. y Vaz-de-Mello, F.Z. (2009). Seasonal and spatial species richness variation of dung beetle (Coleoptera, Scarabaeidae s. str.) in the Atlantic Forest of southeastern Brazil. Revista Brasileira de Entomologia, 53: 607-613. HTTP://www.scielo.br/rbent. 
Hernández, M.I.M., Barreto, P.S.C.S., Costa, V.H., CreaoDuarte, J. y Favila, M.E. (2014). Response of a dung beetle assemblage along a reforestation gradient in Restinga forest. Journal of Insect Conservation, 18: 539546. https://doi.org/10.1007/s10841-014-9645-5.

Hewavithana, D.K., Wijesinghe, M.R., Dangalle, C.D. y Dharmarathna, H.A.S. (2016). Habitat and dung preferences of scarab beetles of the subfamily Scarabaeinae: a case study in a tropical monsoon forest in Sri Lanka. International Journal Tropical Insect Science, 36:97-105. https://doi.org/10.1017/ S1742758416000023.

Holter, P., Scholtz, C.H. y Stenseng, L. (2009). Desert detritivory: nutritional ecology of a dung beetle (Pachysoma glentoni) subsisting on plant litter in arid South African sand dunes. Journal of Arid Environment, 73:1090-1094. jaridenv.2009.04.009.

Holz, S. y Placci, G. (2003). Socioeconomic roots of biodiversity loss in Misiones. En: Galindo-Leal, C. y Gusmão Câmara, I. (eds.). Atlantic Forest of South America: biodiversity status, threats, and outlook. Washington, D.C, U.S.A. pp 207-226.

Izquierdo, A.E., De Angelo, C.D. y Aide, T.M. (2008). Thirty years of human demography and land use change in the Atlantic Forest of Misiones, Argentina: an evaluation of the forest transition model. Ecology Society, 13: 3. https://www.ecologyandsociety.org/vol13/iss2/ $\operatorname{art} 3 /$.

Larsen, T.H. y Forsyth, A. (2005). Trap Spacing and Transect Design for Dung Beetles Biodiversity Studies. Biotropica, 37: 322-325. https://doi.org/10.1111/ j.1744-7429.2005.00042.x.

Marsh, C.J., Louzada, J., Beiroz, W. y Ewers, R.M. (2013). Optimising bait for pitfall trapping of Amazonian dung beetles (Coleoptera: Scarabaeinae). PLoS ONE, 8: e73147. https://doi.org/10.1371/journal. pone. 0073147 .

Monteith, G.B. y Storey, R.I. (1981). The biology of Cephalodesmius, a genus of dung beetles which synthesizes "dung" from plant material (Coleoptera: Scarabaeidae: Scarabaeinae). Memoirs of the Queensland Museum, 20:253-277. https://www.biodiversitylibrary. org/part/154556\#/summary.

Myers, M., Mittermeir, R.A., Mittermeir, C.G., Da Fonseca, G.A.B. y Kent, J. (2000). Biodiversity hotspots for conservation priorities. Nature, 403: 853-858. https:// doi.org/10.1038/35002501.
Myers, N. y Knoll, A.H. (2001). The biotic crisis and the future of evolution. Proceedings of the National Academy of Sciences, 98: 5389-5392. https://doi.org/10.1073/ pnas.091092498.

Neita, J.C. y Escobar, F. (2012). The potential value of agroforestry to dung beetle diversity in the wet tropical forests of the Pacific lowlands of Colombia. Agroforestry Systems, 85: 121-131. https://doi.org/10.1007/s10457011-9445-9.

Nichols, E., Larsen, T., Spector, S., Davis, A.L., Escobar, F., Favila, M. y Vulinec, K. (2007). Global dung beetle response to tropical forest modification and fragmentation: a quantitative literature review and metaanalysis. Biological Conservation, 137: 1-19. https:/doi. org/10.1016/j.biocon.2007.01.023.

Nichols, E., Spector, S., Louzada, J., Larsen, T., Amezquita, S. y Favila, M.E. (2008). Ecological functions and ecosystem services provided by Scarabaeinae dung beetles. Biological conservation, 141: 1461-1474. https:// doi.org/10.1016/j.biocon.2008.04.011.

Nichols, E., Gardner, T.A., Peres, C.A. y Spector, S. (2009). Co-declining mammals and dung beetles: an impending ecological cascade. Oikos, 118: 481-487. https://doi. org/10.1111/j.1600-0706.2009.17268.x.

Nichols, E., Uriarte, M., Bunker, D.E., Favila, M.E., Slade, E.M., Vulinec, K., Larsen, T., Vaz-de-Mello, F.Z., Louzada, J., Naeem, S. y Spector, S.H. (2013). Trait-dependent response of dung beetle populations to tropical forest conversion at local and regional scales. Ecology, 94: 180-189. https://doi.org/10.1890/120251.1.

Novacek, M.J. y Cleland, E.E. (2001). The current biodiversity extinction event: scenarios for mitigation and recovery. Proceedings of the National Academy of Sciences, 98: 5466-5470. https://doi.org/10.1073/ pnas.091093698.

Ocampo, F.C. y Hawks, D.C. (2006). Molecular phylogenetics and evolution of the food relocation behaviour of the dung beetle tribe Euraniini (Coleoptera: Scarabaeidae: Scarabaeinae). Invertebrate Systematics, 20: 557-570.

Oksanen, J., Blanchet, F.G., Friendly, M., Kindt, R., Legendre P., McGlinn, D., Minchin, P.R., O'Hara, R.B., Simpson, G.L., Solymos, P., Stevens, M.H.M., Szoecs, E. y Wagner, H. (2017). vegan: Community Ecology Package. R package version 2.4-3. https:// CRAN.R-project.org/package=vegan 2012 . 
Oliveira-Filho, A.T. y Fontes, I.A.M. (2000). Patterns of floristic differentiation among Atlantic forests in Southeastern Brazil and the Influence of climate. Biotropica, 32: 793-810. https://doi. org/10.1111/j.1744-7429.2000.tb00619.x.

Osberg, D.C., Doube, B.M. y Hanrahan, S.A. (1994). Habitat specificity in African dung beetles. The effect of soil type on the survival of dung beetle immatures (Coleoptera Scarabaeidae). Tropical Zoology, 7: 1-10. https://www.tandfonline.com/doi/pdf/10.1080/03946 975.1994.10539236.

Peyras, M., Vespa, N., Bellocq, M. y Zurita, G. (2012). Quantifying edge effects: the role of habitat contrast and species specialization. Journal of Insect Conservation, 17: 807-820. https://doi.org/10.1007/s10841-013-9563-y.

Philips, K., Pretorius, E. y Scholtz, C. (2004). A phylogenetic analysis of dung beetles (Scarabaeinae: Scarabaeidae): unrolling an evolutionary history. Invertebrate Systematics, 18: 53-88. https://doi. org/10.1071/IS03030.

Quintero, I. y Roslin, T. (2005). Rapid recovery of dung beetle communities following habitat fragmentation in central Amazonia. Ecology, 12: 3303-3311. https://doi. org/10.1890/04-1960.

R Development Core Team. (2017). R: A Language and Environment for Statistical Computing. R Foundation for Statistical Computing, Vienna, Austria. http:// www.R-project.org.

Ribeiro, M.C., Metzger, J.P., Camargo Martensen, A., Ponzoni, F.J. y Hirota, M.M. (2009). The Brazilian Atlantic Forest: How much is left, and how is the remaining forest distributed? Implications for conservation. Biological Conservation, 142: 1141-1153. https://doi.org/10.1016/j.biocon.2009.02.021.

Sánchez-de-Jesús, H., Arroyo-Rodríguez, V., Andresen, E. y Escobar, F. (2016). Forest loss and matrix composition are the major drivers shaping dung beetle assemblages in a fragmented rainforest. Landscape Ecology, 31:843854. https://doi.org/10.1007/s10980-015-0293-2.

Scheffler, P.Y. (2005). Dung beetle (Coleoptera: Scarabaeidae) diversity and community structure across three disturbance regimes in eastern Amazonia. Journal of Tropical Ecology, 21: 9-19. https://doi.org/10.1017/ S0266467404001683.
Schmitte, T., Thorsten, K.F. y Linsenmair, E. (2004). Quinone mixture as attractant for necrophagous dung beetles specialized on dead millipedes. Journal of Chemical Ecology, 30: 731- 740. https://doi. org/10.1023/B:JOEC.0000028428.53797.cb.

Scholtz, C.H., Harrison, J.G. y Grebennikov, V.V. (2004). Dung beetle (Scarabaeus (Pachysoma)) biology and immature stages: reversal to ancestral states under desert conditions (Coleoptera: Scarabaeidae). Biological Journal of the Linnean Society, 83:453-460. https://doi. org/10.1111/j.1095-8312.2004.00389.x.

Slade, E.M., Riutta, T., Roslin, T. y Tuomisto, H.L. (2016). The role of dung beetles in reducing greenhouse gas emissions from cattle farming. Nature, 6: 18140. https://doi.org/10.1038/srep18140.

Sowig, P. (1995). Habitat selection and offspring survival rate in three paracoprid dung beetles: the influence of soil type and soil moisture. Ecography, 18: 147-154. https://doi.org/10.1111/j.1600-0587.1995.tb00335.x.

Spector, S. (2006). Scarabaeine dung beetles (Coleopteran: Scarabaeidae: Scarabaeinae): an invertebrate focal taxon for biodiversity research and conservation. The Coleopterists Bulletin, 60: 71-83. https://doi. org/10.1649/0010-065X(2006)60[71:SDBCSS]2.0. $\mathrm{CO} ; 2$.

Tonelli, M. (2017). Effect of different pasture management on dung beetles communities in a sub-mountainous landscape of central Italy: a multicomponent biodiversity and ecological process analysis. Tesis doctoral. http:// hdl.handle.net/10045/65761.

Tshikae, B.P., Davis, A.L.V. y Scholtz, C.H. (2013). Species richness - Energy relationships and dung beetle diversity across an aridity and trophic resource gradient. Acta Oecología, 49: 71-82. https://doi.org/10.1016/j. actao.2013.02.011.

Tuff, K.T., Tuff, T. y Davies, K.F. (2016). A framework for integrating thermal biology into fragmentation research. Ecology Letter, 19:361-374. https://doi.org/10.1111/ ele. 12579 .

Verdú, J.R., Arellano, L., Numa, C. y Mico, E. (2007a). Roles of endothermy in niche differentiation for ballrolling dung beetles (Coleoptera: Scarabaeidae) along an altitudinal gradient. Ecological Entomology, 32: 544-551. https://doi.org/10.1111/j.1365-2311.2007.00907.x. 
Verdú, J.R., Moreno, C.E., Sánchez-Rojas, G., Numa, C., Galante, E. y Halffter, G. (2007b). Grazing promotes dung beetle diversity in the xeric landscape of a Mexican biosphere reserve. Biological Conservation, 140: 308317. https://doi.org/10.1016/j.biocon.2007.08.015.

Verdú, J.R., Lobo, J.M., Sánchez-Piñero, F., Gallego, B., Numa, C., Lumaret, J.P., Cortez, V., Ortiz, A., Tonelli, M., García-Teba, R.A., Rodriguez, A. y Durán, J. (2018). Ivermectin residues disrupt dung beetle diversity, soil properties and ecosystem functioning: An interdisciplinary field study. Science of the Total Environment, 618: 219-228. https://doi.org/10.1016/j. scitotenv.2017.10.331.
Vulinec, K. (2002). Dung beetles communities and seed dispersal in primary forest and disturbed land in Amazonia. Biotropica, 34: 297-309. https://doi. org/10.1111/j.1744-7429.2002.tb00541.x.

Zaninovich, S.C., Fontana, J.L. y Gatti, M.G. (2016). Atlantic forest replacement by non-native tree plantations: comparing aboveground necromass between native forest and pine plantation ecosystems. Forest Ecology and Management, 363:39-46. https://doi. org/10.1016/j.foreco.2015.12.022.

Zurita, G.A. y Bellocq, M.I. (2012). Bird assemblages in anthropogenic habitats: identifying a suitability gradient for native species in the Atlantic forest. Biotropica, 44: 412-419. https://doi.org/10.1111/ j.1744-7429.2011.00821.x. 


\section{MATERIAL SUPLEMENTARIO}

\section{Tablas}

Tabla MS1. Características generales del bosque nativo y cuatro usos diferentes de la tierra (plantación de pino, parquizado, silvopastoril y potrero para ganadería) en el bosque Atlántico de Argentina.

\section{\begin{tabular}{l|l} 
AMBIENTE & DESCRIPCIÓN GENERAL
\end{tabular}}

Ambiente formado por varios estratos de vegetación (tres a seis): 1) árboles emergentes (> $30 \mathrm{~m}$, principalmente de las familias Rutaceae, Lauraceae y Meliaceae); 2) estrato arbóreo alto (entre los 30 y $25 \mathrm{~m}$ ); 3) estrato arbóreo medio (25 a $20 \mathrm{~m})$; 4) estrato arbóreo bajo

Bosque Nativo (compuesto principalmente por helechos arborescentes y árboles de frutas carnosas), 5) estrato arbustivo y 6) estrato herbáceo (entre los que se encuentran varias especies de helecho) (Cabrera 1971, Campanello et al. 2009). El suelo se caracteriza por ser arenoso y con elevada cantidad de carbono orgánico (Gómez-Cifuentes et al., 2018).

Dedicadas principalmente a la producción de madera y pulpa de celulosa. Se caracterizan

Plantación de pino por una densa cobertura arbórea compuesta por pinos (Pinus taeda) de 7-9 m, con una densidad de 1200 plantas/ha y baja cobertura de sotobosque (Peyras et al. 2012, Zaninovich et al. 2016).

Dedicado a la ganadería. Remanente de bosque nativo abierto compuesto por árboles Parquizado nativos, lianas; y un sotobosque de arbustos, hierbas y bambúes. El suelo se caracteriza por presentar elevados valores de arcilla (Gómez-Cifuentes et al., 2018).

Dedicados a la ganadería y a la producción de madera. Se caracteriza por presentar cobertura arbórea de pino (Pinus spp) de 9-10 m, con una densidad baja de 400 plantas/

Silvopastoril ha. El sotobosque está compuesto principalmente por gramíneas palatables para el ganado y arbustos. El suelo se caracteriza por presentar elevados valores de arcilla (Gómez-Cifuentes et al., 2018).

Dedicado a la ganadería. Áreas abiertas con gramíneas exóticas implantadas para el consumo

Potrero para ganadería del ganado y algunos árboles muy dispersos (Gómez-Cifuentes et al. 2017). El suelo se caracteriza por presentar elevados valores de arcilla (Gómez-Cifuentes et al., 2018).

Tabla MS2. Total de individuos colectados en el bosque nativo (BN) y cuatro usos de la tierra: plantación de pino (PP), parquizado (PZ), silvopastoril (SP) y potrero para ganadería (PG) en el Bosque Atlántico de Argentina. Estudio del nicho trófico llevado a cabo en el 2014. Modificado de Giménez Gómez et al., 2018a.

\begin{tabular}{lcccccc|}
\multicolumn{1}{c}{ ESPECIES } & BN & PP & PZ & SP & PG & TOTAL \\
\hline Anomiopus bonaires & 0 & 0 & 0 & 0 & 1 & 1 \\
Anomiopus wittmery & 0 & 0 & 1 & 0 & 1 & 2 \\
Ateuchus sp. & 0 & 3 & 1 & 0 & 0 & 4 \\
Canthidium aff. Breve & 0 & 7 & 3 & 0 & 0 & 10 \\
Canthidium cavifrons & 1 & 4 & 0 & 0 & 0 & 5 \\
Canthidium dispar & 14 & 1 & 0 & 1 & 0 & 16 \\
Canthidium hyla & 1 & 0 & 0 & 0 & 0 & 1 \\
Canthidium moestum & 0 & 2 & 2 & 2 & 0 & 6 \\
Canthidium nobile & 0 & 0 & 0 & 2 & 0 & 2 \\
Canthidium sp 2. & 0 & 0 & 0 & 5 & 0 & 5 \\
Canthidium sp 3. & 2 & 0 & 3 & 0 & 0 & 5
\end{tabular}




\begin{tabular}{|c|c|c|c|c|c|c|}
\hline ESPECIES & BN & PP & PZ & SP & PG & TOTAL \\
\hline Canthidium sp 4. & 0 & 0 & 0 & 0 & 1 & 1 \\
\hline Canthon conformis & 6 & 22 & 382 & 211 & 15 & 636 \\
\hline Canthon histrio & 0 & 10 & 202 & 22 & 0 & 234 \\
\hline Canthon podagricus & 0 & 0 & 1 & 0 & 150 & 151 \\
\hline Canthon quinquemaculatus & 193 & 467 & 914 & 343 & 25 & 1942 \\
\hline Canthon smaragdulus & 7 & 1 & 0 & 0 & 0 & 8 \\
\hline Coprophanaeus cyanescens & 14 & 41 & 27 & 37 & 4 & 123 \\
\hline Coprophanaeus saphirinus & 102 & 19 & 56 & 39 & 0 & 216 \\
\hline Deltochilum brasiliensis & 1 & 0 & 2 & 0 & 0 & 3 \\
\hline Deltochilum furcatum & 1 & 7 & 1 & 1 & 0 & 10 \\
\hline Deltochilum icaroides & 1 & 9 & 0 & 2 & 0 & 12 \\
\hline Deltochilum aff. komareki & 86 & 460 & 437 & 188 & 14 & 1185 \\
\hline Deltochilum morbillosum & 7 & 13 & 0 & 0 & 0 & 20 \\
\hline Dichotomius carbonarus & 1 & 0 & 0 & 0 & 0 & 1 \\
\hline Dichotomius depressicollis & 1 & 0 & 0 & 3 & 0 & 4 \\
\hline Dichotomius nisus & 0 & 1 & 0 & 0 & 54 & 55 \\
\hline Dichotomius sericeus & 278 & 588 & 871 & 377 & 14 & 2128 \\
\hline Dichotomius sp 1. & 0 & 0 & 1 & 0 & 0 & 1 \\
\hline Eurysternus aeneus & 0 & 0 & 0 & 1 & 0 & 1 \\
\hline Eurysternus caribaeus & 18 & 167 & 28 & 5 & 0 & 218 \\
\hline Eurysternus parallelus & 6 & 2 & 37 & 2 & 0 & 47 \\
\hline Eutrichillum hirsutum & 0 & 0 & 0 & 0 & 5 & 5 \\
\hline Onthophagus aff. buculus & 0 & 1 & 0 & 0 & 0 & 1 \\
\hline Onthophagus catharinensis & 1 & 2 & 0 & 0 & 0 & 3 \\
\hline Onthophagus tristis & 0 & 6 & 0 & 0 & 0 & 6 \\
\hline Scybalocanthon nigriceps & 2 & 0 & 0 & 0 & 0 & 2 \\
\hline Trichillum sp 1. & 1 & 0 & 0 & 0 & 0 & 1 \\
\hline Trichillum sp 2. & 1 & 0 & 0 & 0 & 0 & 1 \\
\hline Uroxys dilaticollis & 0 & 3 & 0 & 0 & 0 & 3 \\
\hline Uroxys sp 1. & 7 & 2 & 0 & 0 & 0 & 9 \\
\hline Uroxys sp 2 & 6 & 0 & 0 & 0 & 0 & 6 \\
\hline Uroxys sp 3. & 1 & 0 & 0 & 0 & 0 & 1 \\
\hline Uroxys thoracalis & 0 & 302 & 0 & 0 & 0 & 302 \\
\hline Total & 759 & 2140 & 2969 & 1241 & 284 & 7393 \\
\hline
\end{tabular}


Tabla MS3. Total de individuos colectados en el bosque nativo (BN) y cuatro usos de la tierra: plantación de pino (PP), parquizado (PZ), silvopastoril (SP) y potrero para ganadería (PG) en el Bosque Atlántico de Argentina. Estudio de diversidad llevado a cabo en el 2016. Modificado de Giménez Gómez et al., 2018b.

\begin{tabular}{|c|c|c|c|c|c|c|}
\hline ESPECIES & $\mathbf{B N}$ & PP & $\mathbf{P Z}$ & SP & PG & Total \\
\hline Ateuchus sp. & 0 & 1 & 2 & 0 & 0 & 3 \\
\hline Canthidium bituberculatum & 0 & 0 & 3 & 0 & 1 & 4 \\
\hline Canthidium cavifrons & 0 & 0 & 3 & 0 & 0 & 3 \\
\hline Canthidium dispar & 5 & 2 & 0 & 0 & 0 & 7 \\
\hline Canthidium hyla & 1 & 1 & 0 & 2 & 9 & 13 \\
\hline Canthidium lucidum & 0 & 3 & 1 & 0 & 0 & 4 \\
\hline Canthidium nobile & 0 & 0 & 4 & 1 & 0 & 5 \\
\hline Canthidium sp. & 33 & 0 & 12 & 4 & 0 & 49 \\
\hline Canthon conformis & 1 & 37 & 718 & 335 & 16 & 1107 \\
\hline Canthon curvodilatus & 0 & 0 & 0 & 0 & 13 & 13 \\
\hline Canthon histrio & 1 & 2 & 440 & 8 & 0 & 451 \\
\hline Canthon paraguayanus & 0 & 0 & 6 & 0 & 0 & 6 \\
\hline Canthon podagricus & 0 & 0 & 0 & 0 & 290 & 290 \\
\hline Canthon quinquemaculatus & 409 & 269 & 2933 & 807 & 14 & 4432 \\
\hline Chalcocopris hesperus & 25 & 0 & 0 & 0 & 0 & 25 \\
\hline Coprophanaeus cyanescens & 144 & 89 & 134 & 90 & 12 & 469 \\
\hline Coprophanaeus saphirinus & 95 & 11 & 83 & 61 & 0 & 250 \\
\hline Deltochilum brasiliensis & 21 & 0 & 0 & 0 & 0 & 21 \\
\hline Deltochilum furcatum & 29 & 6 & 7 & 14 & 0 & 56 \\
\hline Deltochilum icaroides & 0 & 0 & 2 & 0 & 0 & 2 \\
\hline Deltochilum aff. komareki & 135 & 237 & 781 & 797 & 10 & 1960 \\
\hline Deltochilum morbillosum & 4 & 1 & 0 & 0 & 0 & 5 \\
\hline Dichotomius aff. fissus & 0 & 3 & 1 & 0 & 0 & 4 \\
\hline Dichotomius carbonarius & 16 & 0 & 129 & 86 & 7 & 238 \\
\hline Dichotomius depresicollis & 4 & 18 & 3 & 5 & 0 & 30 \\
\hline Dichotomius mormon & 17 & 12 & 34 & 75 & 0 & 138 \\
\hline Dichotomius nisus & 0 & 0 & 0 & 34 & 163 & 197 \\
\hline Dichotomius sericeus & 296 & 331 & 529 & 677 & 5 & 1838 \\
\hline Eurysternus aeneus & 0 & 0 & 3 & 1 & 1 & 5 \\
\hline Eurysternus caribaeus & 377 & 865 & 160 & 130 & 0 & 1532 \\
\hline Eurysternus howdeni & 1 & 0 & 0 & 0 & 0 & 1 \\
\hline Eurysternus parallelus & 152 & 33 & 332 & 36 & 2 & 555 \\
\hline Eutrichillum hirsutum & 1 & 67 & 49 & 95 & 305 & 517 \\
\hline Ontherus erosioides & 0 & 0 & 1 & 0 & 0 & 1 \\
\hline Ontherus sulcator & 0 & 4 & 13 & 22 & 32 & 70 \\
\hline Ontophagus aff. búculus & 0 & 0 & 2 & 5 & 12 & 19 \\
\hline Ontophagus catharinensis & 68 & 21 & 6 & 1 & 0 & 96 \\
\hline Ontophagus sp. & 0 & 0 & 0 & 1 & 0 & 1 \\
\hline
\end{tabular}




\begin{tabular}{lcccccc}
\multicolumn{1}{c}{ ESPECIES } & BN & PP & PZ & SP & PG & Total \\
\hline Ontophagus tristis & 21 & 132 & 2 & 5 & 1 & 160 \\
Phanaeus splendidulus & 2 & 0 & 0 & 0 & 0 & 2 \\
Scybalocanthon nigriceps & 12 & 0 & 0 & 0 & 0 & 12 \\
Trichillum externepunctatum & 0 & 0 & 0 & 0 & 5 & 5 \\
Trichillum hesper & 0 & 0 & 0 & 0 & 18 & 18 \\
Uroxys dilaticollis & 3 & 19 & 0 & 0 & 0 & 22 \\
Uroxys epipleuralis & 0 & 0 & 1 & 0 & 0 & 1 \\
Uroxys sp. & 2 & 0 & 1 & 0 & 0 & 3 \\
Uroxys thoracalis & 0 & 70 & 0 & 0 & 0 & 70 \\
\hline Total & $\mathbf{1 8 7 5}$ & $\mathbf{2 2 3 4}$ & $\mathbf{6 3 9 5}$ & $\mathbf{3 2 9 2}$ & $\mathbf{9 1 6}$ & $\mathbf{1 4 7 1 2}$
\end{tabular}

Tabla MS4. Resultados de similitud promedio (a través de PERMANOVA) entre el bosque nativo y cuatro usos de la tierra en el bosque Atlántico de Argentina. $\mathrm{BN}=$ bosque nativo, $\mathrm{PP}=$ plantación de pino, $\mathrm{PZ}=$ parquizado, $\mathrm{SP}=$ silvopastoril y $\mathrm{PG}=$ potrero para ganadería. ${ }^{*} \mathrm{p}<0.05,{ }^{* *} \mathrm{p}<0.01$. Modificado de Giménez Gómez et al., 2018b.

\begin{tabular}{c|c|c|c|c} 
& BN & PP & PZ & SP \\
\hline BN & - & - & - & - \\
\hline PP & $63.262^{* *}$ & - & & - \\
\hline PZ & $59.655^{* *}$ & $63.127^{*}$ & - & - \\
\hline SP & $60.144^{* *}$ & $65.862^{* *}$ & 75.339 & - \\
\hline PG & $15.470^{* *}$ & $22.871^{*}$ & $26.879^{* *}$ & $30.135^{*}$
\end{tabular}



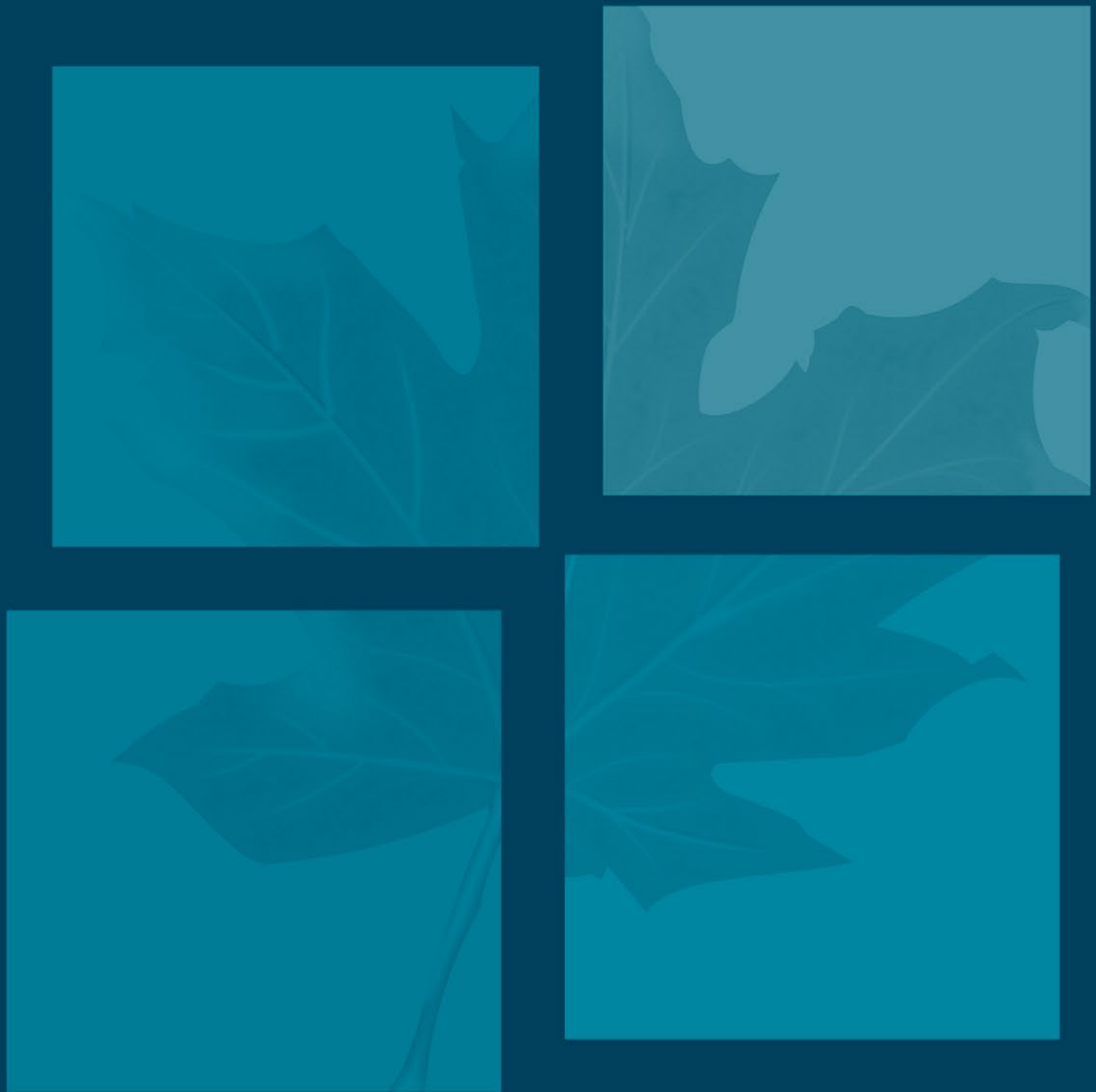